\title{
THE PROOF-THEORETIC ANALYSIS OF TRANSFINITELY ITERATED QUASI LEAST FIXED POINTS
}

\author{
DIETER PROBST
}

\begin{abstract}
The starting point of this article is an old question asked by Feferman in his paper on Hancock's conjecture [6] about the strength of $I D_{1}^{*}$. This theory is obtained from the well-known theory $I D_{1}$ by restricting fixed point induction to formulas that contain fixed point constants only positively. The techniques used to perform the proof-theoretic analysis of $I D_{1}^{*}$ also permit to analyze its transfinitely iterated variants $I D_{\alpha}^{*}$. Thus, we eventually know that ||$\widehat{D}_{\alpha}|=| I D_{\alpha}^{*} \mid$.
\end{abstract}

§1. Introduction. The theories $I D_{\alpha}$ of iterated inductive definitions formalize hierarchies of least (definable) fixed points. In the past years, these theories have been exhaustively studied and their proof-theoretic analysis has been carried out a long time ago, (cf. Buchholz et al. [3]). Also their metapredicative relatives $\widehat{I D}_{\alpha}$, that speak about hierarchies of (not necessary least) fixed points are well understood by now. The proof-theoretic ordinal of $\widehat{\mathrm{ID}}_{1}$ is due to Aczel [1], who used a recursion theoretic argument, nowadays known as Aczel's trick, to embed $\widehat{\mathrm{ID}}_{1}$ into $\Sigma_{1}^{1}-\mathrm{AC}$. The theories $\widehat{\mathbb{D D}}_{n}$ of $n$-times iterated inductive definitions have been analyzed by Feferman in connection with Handcock's conjecture in [6]. The proof-theoretic analysis of $\widehat{\mathrm{ID}}_{\alpha}$ has been carried out in all details by Jäger, Kahle, Setzer and Strahm [9].

Some problems however, have remained unsolved: In the theories $\widehat{I D}_{\alpha}$, induction on fixed points is dropped completely. It is natural to study theories, where fixed point induction is only restricted. Kreisel pointed out in [11], that "an inductive definition tells you what is in $\mathrm{P}^{\mathscr{A}}$ not what is not in $\mathrm{P}^{\mathscr{A}}$ ". As mentioned in Feferman [6], this motivated to consider restricted versions of $I D_{1}$ such as $I D_{1}^{*}$, a theory credited to H. Friedman where the scheme for proof by induction on fixed points is restricted to formulas that contain fixed point constants only positively. The question for a sharp upper bound is raised loc. cit. No answer to this question has yet been published, although partial results have been attained: If the fixed point axioms of $I D_{1}^{*}$ are restricted to so-called accessibility inductive definitions, then the resulting theory $I_{1}^{*}(\mathscr{C E})$ can be embedded in $\Sigma_{1}^{1}-\mathrm{DC}$ as sketched by Feferman in [6]. There, it is also stated that Friedman [8] introduced the theory ID* and showed that its ordinal is bounded by $\alpha_{1}$, where $\alpha_{0}:=\varepsilon_{0}$ and $\alpha_{n+1}:=\varphi \alpha_{n} 0$. Further, upper bounds for

Received June 15, 2004.

Key words and phrases. Fixed points; Iteration; Pseudo-hierarchies.

The author is supported by the Swiss National Science Foundation.

(C) 2006, Association for Symbolic Logic $0022-4812 / 06 / 7103-0001 / \$ 3.60$ 
the theories $I D_{n}^{*}$ are computed by Cantini [4]. Thereby the two common ordinal measures for a theory $T$ of inductive definitions are considered: The proof-theoretic ordinal $|\mathrm{T}|$, i.e., the least ordinal $\alpha$ such that for no primitive recursive well-ordering $\prec$ of ordertype $\alpha$ the well-orderedness of $\prec$ is provable within T, and alternatively, the least stage $\|\mathrm{T}\|$ not provable in T. The $\xi$ th stage $I_{\xi}^{\mathscr{E}}$ of an inductive definition $\mathscr{C}$ is given by $\left\{x: \mathscr{E}\left(\bigcup_{\eta<\xi} I_{\eta}^{\mathscr{E}}, x\right)\right\}$, and a stage $\alpha$ is called provable if there is an inductive definition $\mathscr{A}$ and an $m \in \mathbb{N}$ with $\mathrm{T} \vdash m \in \mathrm{P}^{\mathscr{A}}$, such that $\alpha \leq \min \left\{\beta: m \in I_{\beta}^{\mathscr{A}}\right\}$. In [4], Cantini proves that $\left|\mathrm{ID}_{n}^{*}\right| \leq \alpha_{2^{n}}$ and $\left\|\mid \mathrm{ID}_{n}^{*}\right\| \leq \alpha_{2^{n}-1}$ for $n>0$ and conjectures, like Feferman in [6], $\left|I D_{n}^{*}\right|=\alpha_{n}=\left\|I D_{n}^{*}\right\|$ to hold true. We point out that for impredicative theories these two ordinal assignments usually coincide, whereas in (meta-)predicative theories they usually differ: $\left|\mathrm{ID}_{n}\right|=\left\|\mathrm{ID}_{n}\right\|$ is the case, however $\left\|\widehat{I D}_{n}\right\|=\alpha_{n-1}$ only unfolds to $\left|\widehat{I D}_{n}\right|=\alpha_{n}$. In respect thereof, the theories $\mathrm{ID}_{n}^{*}$ take an exceptional position, as ||$D_{n}^{*}|=| \mid I D_{n}^{*} \|$ follows from our wellordering proof.

This article provides the proof-theoretic ordinals of the theories $\mid \mathrm{D}_{\alpha}^{*}$ and $\mid \mathrm{D}_{\alpha}^{*} \uparrow$, a variant where also induction on the natural numbers is restricted to formulas that contain fixed point constants only positively. In the first sections we treat the case $I_{1}^{*}$. Thereto we present a new embedding of $\widehat{I D}_{1}$ into $\Sigma_{1}^{1}-A C$ that extends to an embedding of $I D_{1}^{*}$ into $\Sigma_{1}^{1}-D C$. This embedding relies on the following two observations: Already $\Sigma_{1}^{1}-A C$ proves that for an operator form $\mathscr{A}\left(\mathrm{P}^{+}, \mathrm{p}\right)$, the standard $\Pi_{1}^{1}$ definition of the fixed point, namely the intersection of all sets $X$ satisfying $\forall x[\mathscr{A}(X, x) \rightarrow x \in X]$, is a fixed point of the operator defined by $\mathscr{A}$. Moreover, $\Sigma_{1}^{1}-\mathrm{DC}$ proves that this is indeed the least $\Pi_{1}^{1}$-definable fixed point.

To demonstrate the power of restricted fixed point induction, we give wellordering proofs for $I D_{\alpha}^{*}$ and $I D_{\alpha}^{*} \uparrow$. The general idea is the same as in [9] or [7], but things are simpler and the proofs are carried out in $\mid D_{\alpha}^{*}$ and $I D_{\alpha}^{*}\lceil$ themselves. Section 7 is devoted to the upper bounds. In [15], Rüede has developed and analyzed semi-formal systems to treat theories $M_{\alpha}$, formalizing transfinite hierarchies of models of $\Sigma_{1}^{1}-A C$. To embed the theories $I D_{\alpha}^{*}$ and $\left.\mid D_{\alpha}^{*}\right\rceil$ into such systems, we require uniform hierarchies of models of $\Sigma_{1}^{1}-D C$. Towards this, we extend $M_{\alpha}$ to $M_{\alpha}^{\dagger}$, by an axiom claiming that transfinite induction for $\left|M_{\alpha}\right|$, the proof-theoretic ordinal of $\mathrm{M}_{\alpha}$, fails. According to Jäger and Probst [10], this extension is conservative. In $\mathrm{M}_{\alpha}^{\dagger}$, the technique of pseudo-hierarchies can be applied to construct the required hierarchies. Rüede's results then yield sharp upper bounds.

§2. The theories $\mid D_{1}^{*}$ and $\left.\mid D_{1}^{*}\right\rceil$. Let $L_{1}$ be a standard language of first order arithmetic that includes number variables $a, b, c, d, e, u, v, w, x, y, z, \ldots$ and function and relation symbols for all primitive recursive functions and relations. In particular, we have a unary relation symbol $\mathrm{N}$ for the natural numbers. Moreover, we have unary relation symbols $U$ and $V$ that are required for technical reasons. Since we consider Tait-style calculi in the sequel, we use the symbol $\sim$ for forming negative literals, and define the negation $\neg A$ of a formula $A$ of $L_{1}$ or some language containing $L_{1}$ by making use of De Morgan's laws and the law of double negation. For $\mathrm{U}(t)$ and $\sim \mathrm{U}(t)$ we write $t \in \mathrm{U}$ and $t \notin U$.

Towards the formulation of $I D_{1}^{*}$, we extend $L_{1}$ by fresh unary relation symbols $\vec{P}, \vec{Q}$ and a fresh number constant $p$, which serve as placeholders. Then, a P-positive formula of $L_{1}(P, \vec{Q}, p)$, the extension of $L_{1}$ by $P, \vec{Q}$ and $p$, is called an inductive 
operator form, and we let $\mathscr{A}$ range over such forms. For sets $\vec{Y}$ and numbers $\vec{y}$, an operator form $\mathscr{A}(\mathrm{P}, \overrightarrow{\mathrm{Q}}, \mathrm{q}, \vec{u})$ defines an operator on the powerset of the natural numbers, namely

$$
F_{\vec{Y}, \vec{y}}^{\mathscr{A}}(X):=\{x: \mathscr{A}(X, \vec{Y}, x, \vec{y})\} .
$$

Next, we add to the first order language $L_{1}$ a fixed point constant $P^{\mathscr{A}}$ for each inductive operator form $\mathscr{A}$ of $L_{1}(P, p)$ without free variables, and denote this new language by $L_{F i x}$. Technically, we treat fixed point constants as unary relation symbols, but write $t \in \mathrm{P}^{\mathscr{A}}$ instead of $\mathrm{P}^{\mathscr{x}}(t)$. The formulas $A, B, C, \ldots$ and the number terms $r, s, t, \ldots$ of $\mathrm{L}_{\text {Fix }}$ are defined in the expected way and the formulas of $\mathrm{L}_{\mathrm{Fix}}^{+}$are the formulas of $\mathrm{L}_{\mathrm{Fix}}$ that contain fixed point constants only positively.

The axioms of $I D_{1}^{*}$ consist of the axioms of PA without induction, complete induction along the natural numbers for all formulas of $L_{F i x}$ as well as the following two fixed point axioms: For all inductive operator forms $\mathscr{A}(\mathrm{P}, \mathrm{p})$ without free variables, we have

$$
\forall x\left[\mathscr{A}\left(\mathrm{P}^{\mathscr{A}}, x\right) \leftrightarrow x \in \mathrm{P}^{\mathscr{A}}\right]
$$

and for all inductive operator forms $\mathscr{A}(\mathrm{P}, \mathrm{p}), \mathscr{A}_{1}(\mathrm{P}, \mathrm{p}), \ldots, \mathscr{A}_{n}(\mathrm{P}, \mathrm{p})$ without free variables, and each $\vec{P}$-positive formula $B(\vec{P}, p, \vec{u})$ of $L_{1}(\vec{P}, p)$, we have

$$
\begin{aligned}
&\left(\mathrm{IND}_{\mathrm{FIX}}^{+}\right) \quad \forall x\left[\mathscr{A}\left(\left\{z: B\left(\mathrm{P}^{\mathscr{A}}, z, \vec{y}\right)\right\}, x\right) \rightarrow B\left(\mathrm{P}^{\overrightarrow{\mathscr{A}}}, x, \vec{y}\right)\right] \rightarrow \\
& \forall x\left[x \in \mathrm{P}^{\mathscr{A}} \rightarrow B\left(\mathrm{P}^{\mathscr{A}}, x, \vec{y}\right)\right] .
\end{aligned}
$$

Note that we wrote $\mathrm{P}^{\overrightarrow{\mathscr{A}}}$ for the string $\mathrm{P}^{\mathscr{A}_{1}}, \ldots, \mathrm{P}^{\mathscr{X}_{n}}$ and that $\mathscr{A}$ may be syntactically identical to some $\mathscr{A}_{i}$. The axiom (FIX) asserts that $\mathrm{P}^{\mathscr{A}}$ is indeed a fixed point of the operator $F^{\mathscr{A}}$ and $\left(\mathrm{IND}_{\mathrm{FIX}}^{+}\right)$is the scheme for proof by induction on $\mathrm{P}^{\mathscr{A}}$ restricted to formulas of $\mathrm{L}_{\mathrm{Fix}}^{+}$. Finally, $\mid \mathrm{ID}_{1}^{*} \uparrow$ denotes the theory where also complete induction along the natural numbers is restricted to formulas of $\mathrm{L}_{\mathrm{Fix}}^{+}$, and $\widehat{\mathrm{ID}}_{1}$ is $\mathrm{ID}_{1}^{*}$ without $\left(\mathrm{IND}_{\mathrm{FIX}}^{+}\right)$.

In this article, we make use of the term proof-theoretic ordinal. For theories $T$ that are formulated in a language comprising $L_{1}$, the proof-theoretic ordinal of $T$ can be defined in the following way: We set

$$
\begin{aligned}
\operatorname{Prog}_{\prec}(Z) & :=\forall u(\forall v \prec u)(v \in Z \rightarrow u \in Z), \\
\operatorname{TI}_{\prec}(Z, t) & :=\operatorname{Prog}_{\prec}(Z) \rightarrow(\forall u \prec t)(u \in Z),
\end{aligned}
$$

and call an ordinal $\alpha$ provable in $T$, if there exists a primitive recursive well-ordering $\prec$ such that $\mathrm{T} \vdash \mathrm{TI}_{\prec}(\mathrm{U}, \alpha)$. Any ordinal that is not provable in $\mathrm{T}$ is called an upper bound of $\mathrm{T}$ and the least ordinal that is not provable in $\mathrm{T}$ is then the proof-theoretic ordinal of $T$, denoted by $|T|$.

§3. A new embedding of $\widehat{I D}_{1}$ into $\Sigma 1$-AC. The standard embedding of $\widehat{I D}_{1}$ into $\Sigma_{1}^{1}-A C$ is due to Aczel [1]. He makes use of a universal $\Sigma_{1}^{1}$ formula and a standard diagonalization argument to find a $\Sigma_{1}^{1}$ definable solution for each fixed point constant $P^{\mathscr{A}}$ respecting (FIX). Of course, there is no chance to prove that such a solution is minimal with respect to classes definable by $L_{\mathrm{Fix}}^{+}$formulas. Bearing such a minimality condition in mind, the most natural way to interpret a fixed point constant $P^{\mathscr{A}}$ is to take its $\Pi_{1}^{1}$ definition, i.e., the intersection of all sets satisfying 
$F^{\mathscr{A}}(X) \subseteq X$. This is indeed in accord with axiom (FIX). Surprisingly enough, the compact proof of this fact has not yet been discovered. Prior to its presentation, we specify the language and axioms of the theories involved, and briefly recap Aczel's argument.

The theories $\Sigma_{1}^{1}-A C$ and $\Sigma_{1}^{1}-A C_{0}$ are formulated in the language $L_{2}$ that canonically extends our language $L_{1}$ to a language of second order arithmetic by set variables $U, V, W, X, Y, Z, \ldots$, a symbol $\in$ to denote elementhood and quantifiers for second order variables. Note, that we write $t \notin X$ for $\sim(t \in X)$. The number terms of $L_{2}$ are the number terms of $L_{1}$. Formulas of $L_{2}$ that do not contain bounded set variables are called arithmetical. $\mathrm{L}_{2}$ formulas of the form $\exists X A(X)$, where $A$ is arithmetical, are called $\Sigma_{1}^{1}$ formulas, and formulas of the form $\neg B$, where $B$ is $\Sigma_{1}^{1}$, are called $\Pi_{1}^{1}$ formulas. The class of $\Pi$ formulas of $L_{2}$ is the smallest class containing the arithmetical formulas of $L_{2}$ that is closed under conjunction, disjunction, number quantification and universal set quantification. If $A$ is a $\Pi$ formula of $\mathrm{L}_{2}$, then $\neg A$ is a $\Sigma$ formula of $\mathrm{L}_{2}$. Arithmetical formulas of $\mathrm{L}_{2}$ where all number quantifiers appear in the context $(\forall x<t)$ and $(\exists x<t)$ are called $\Delta_{0}^{0}$.

In the sequel, we make use of the usual coding machinery: $\langle\ldots\rangle$ is a standard primitive recursive function for forming $n$-tuples $\left\langle t_{0}, \ldots, t_{n-1}\right\rangle$, so-called sequence numbers; $(t)_{i}$ is the $i$ th component of (the sequence coded by) $t$, if $i$ is less than the length $\operatorname{lh}(t)$ of $t$; i.e., $(t)_{i}=t_{i}$ for all $0 \leq i \leq n-1$, provided that $t=\left\langle t_{0}, \ldots, t_{n-1}\right\rangle$. Further, we write $s \in(X)_{t}$ for $\langle s, t\rangle \in X$, and $X=Y$ is to abbreviate the formula $\forall x[x \in X \leftrightarrow x \in Y]$.

Besides the usual axioms of classical logic with equality in the first sort and axioms for the primitive recursive functions and relations, the theory $\Sigma_{1}^{1}-A C$ comprises the schema of complete induction on the natural numbers for all formulas of $\mathrm{L}_{2}$, arithmetical comprehension (ACA), and for all $\Sigma_{1}^{1}$ formulas $A(U, u)$ an axiom

$$
\forall x \exists X A(X, x) \rightarrow \exists Y \forall x A\left((Y)_{x}, x\right) .
$$

$\Sigma_{1}^{1}-\mathrm{AC}_{0}$ is $\Sigma_{1}^{1}-\mathrm{AC}$ with the schema of complete induction on the natural numbers restricted to sets.

Below we observe that in a theory comprising $\Sigma_{1}^{1}-A C_{0}$, we do not have to distinguish between $\Pi$ and $\Pi_{1}^{1}$ formulas of $L_{2}$. Of course, this applies also to the dual classes of $\Sigma_{1}^{1}$ and $\Sigma$ formulas.

LEMMA 3.1. For each $\Pi$ formula $C$ of $\mathrm{L}_{2}$ there is a $\Pi_{1}^{1}$ formula $C^{\prime}$ of $\mathrm{L}_{2}$ containing the same free variables as $C$, such that $\Sigma_{1}^{1}-A C$ proves: $C \leftrightarrow C^{\prime}$.

Aczel's embedding of $\widehat{\mathrm{ID}}_{1}$ into $\Sigma_{1}^{1}-\mathrm{AC}$ relies on this observation and the fact, that there exists a universal $\Sigma_{1}^{1}$ formula $E(u, v, w)$ of $L_{2}$ : For each $\Sigma$ formula $B(u, v)$ of $\mathrm{L}_{2}$, there exists an $e \in \mathbb{N}$ such that

$$
\Sigma_{1}^{1}-\mathrm{AC}_{0} \vdash B(x, y) \leftrightarrow E(\bar{e}, x, y),
$$

where $\bar{e}$ denotes the constant for the natural number $e$. This means in particular, that for a given operator form $\mathscr{A}$ of $\mathrm{L}_{1}(\mathrm{P}, \mathrm{p})$, there is an $e_{\mathscr{A}} \in \mathbb{N}$ such that

$$
\Sigma_{1}^{1}-\mathrm{AC}_{0} \vdash \mathscr{A}(\{z: E(x, x, z)\}, y) \leftrightarrow E\left(\bar{e}_{\mathscr{A}}, x, y\right) .
$$


Letting $C(u)$ be the $\Sigma_{1}^{1}$ formula $E\left(\bar{e}_{\mathscr{A}}, \bar{e}_{\mathscr{A}}, u\right)$, then $\Sigma_{1}^{1}-\mathrm{AC}_{0}$ proves:

$$
\begin{aligned}
\mathscr{A}(\{z: C(z)\}, x) & \leftrightarrow \mathscr{A}\left(\left\{z: E\left(\bar{e}_{\mathscr{A}}, \bar{e}_{\mathscr{A}}, z\right)\right\}, x\right) \\
& \leftrightarrow E\left(\bar{e}_{\mathscr{A}}, \bar{e}_{\mathscr{A}}, x\right) \\
& \leftrightarrow C(x) .
\end{aligned}
$$

If we translate an $\mathrm{L}_{\mathrm{Fix}}$ formula $B$ to an $\mathrm{L}_{2}$ formula $\tilde{B}$ by substituting each subformula of $B$ of the form $t \in \mathrm{P}^{\mathscr{A}}$ by the $\Sigma_{1}^{1}$ formula $E\left(\bar{e}_{\mathscr{A}}, \bar{e}_{\mathscr{A}}, t\right)$, then we obtain the following theorem:

THEOREM 3.2 (Aczel). For every $\mathrm{L}_{\mathrm{Fix}}$ formula $B$ the following holds:

$$
\widehat{\mathrm{ID}}_{1} \vdash B \Longrightarrow \Sigma_{1}^{1}-\mathrm{AC} \vdash \tilde{B} \text {. }
$$

The canonic candidate to interpret the fixed point constant $\mathrm{P}^{\mathscr{A}}$, however, is the intersection of all $\mathscr{A}$-closed sets, namely the $\Pi_{1}^{1}$-definable class

$$
\operatorname{Fix}^{\mathscr{A}}:=\bigcap\left\{X: F^{\mathscr{A}}(X) \subseteq X\right\} .
$$

Of course, we cannot prove in $\Sigma_{1}^{1}-\mathrm{AC}$ that $\mathrm{Fix}^{\mathscr{A}}$ is a set, yet $F^{\mathscr{A}}\left(\mathrm{Fix}^{\mathscr{A}}\right) \subseteq \mathrm{Fix}$ is still immediate: For all $\mathscr{A}$-closed sets $X$, the positivity of the operator form $\mathscr{A}$ yields $F^{\mathscr{A}}\left(\mathrm{Fix}^{\mathscr{A}}\right) \subseteq F^{\mathscr{A}}(X) \subseteq X$. For the other direction, though, we can no longer argue that $F^{\mathscr{A}}\left(\mathrm{Fix}^{\mathscr{A}}\right)$ is $\mathscr{A}$-closed, and therefore a superset of Fix ${ }^{\mathscr{A}}$. To show that $\Sigma_{1}-\mathrm{AC}_{0}$ proves $\mathrm{Fix}^{\mathscr{A}} \subseteq F^{\mathscr{A}}\left(\mathrm{Fix}^{\mathscr{A}}\right)$, a more refined argument is required.

We prove $F^{\mathscr{A}}\left(\mathrm{Fix}^{\mathscr{A}}\right)=\mathrm{Fix}^{\mathscr{A}}$ in a sightly more general context. For an operator form $\mathscr{A}(\mathrm{P}, \overrightarrow{\mathrm{Q}}, \mathrm{p}, \vec{u})$ of $\mathrm{L}_{1}(\mathrm{P}, \overrightarrow{\mathrm{Q}}, \mathrm{p})$ we set

$$
\begin{aligned}
\mathrm{Cl}_{\vec{Y}, \vec{y}}^{\mathscr{A}}(X) & :=\forall x(\mathscr{A}(X, \vec{Y}, x, \vec{y}) \rightarrow x \in X), \\
\operatorname{Fix}_{\vec{Y}, \vec{y}}^{\mathscr{A}} & :=\left\{x: \forall X\left[\mathrm{Cl}_{\vec{Y}, \vec{y}}^{\mathscr{A}}(X) \rightarrow x \in X\right]\right\} .
\end{aligned}
$$

Often, we do not explicitly indicate the parameters in the operator form $\mathscr{A}$, and write $\mathrm{Cl}^{\mathscr{A}}(X)$, Fix ${ }^{\mathscr{A}}$ and $F^{\mathscr{A}}$ instead of $\mathrm{Cl}_{\vec{Y}, \vec{y}}^{\mathscr{A}}(X)$, Fix $\vec{Y}_{\vec{Y}, \vec{y}}^{\mathscr{A}}$ and $F_{\vec{Y}, \vec{y}}^{\mathscr{A}}$. The context provides always enough information to identify the dropped parameters. Below, we prove within $\Sigma_{1}^{1}-A C_{0}$ that $\mathrm{Fix}_{\vec{Y}, \vec{y}}^{\mathscr{A}}$ is a fixed point of the operator $F_{\vec{Y}, \vec{y}}^{\mathscr{A}}$. The direction from right to left is again immediate. For the other direction, the following lemma almost handles the job.

LEMMA 3.3 (Separation Lemma). For all operator forms $\mathscr{A}$ of $\mathrm{L}_{1}(\mathrm{P}, \overrightarrow{\mathrm{Q}}, \mathrm{p})$ and each arithmetical, $U$-positive formula $B(U, u)$ of $\mathrm{L}_{2}, \Sigma_{1}^{1}-\mathrm{AC}_{0}$ proves:

$$
\neg B\left(\mathrm{Fix}_{\vec{Y}, \vec{y}}^{\mathscr{A}}, x\right) \rightarrow \exists X\left[\mathrm{Cl}_{\vec{Y}, \vec{y}}^{\mathscr{A}}(X) \wedge \neg B(X, x)\right] .
$$

Proof. We prove the lemma by induction on the build-up of the formula $B(U, u)$. If $U$ does not occur in $B$ there is nothing to prove, and if $B$ is the formula $t \in U$, then the claim follows from the definition of Fix ${ }^{\mathscr{A}}$. If $B$ is a conjunction or a disjunction, a similar argument applies as in the cases treated below.

(i) $B(U, u)$ is of the form $\exists y B_{1}(U, u, y)$. Assume $\forall y \neg B_{1}\left(\mathrm{Fix}^{\mathscr{}}, x, y\right)$. The I.H. reads

$$
\neg B_{1}\left(\mathrm{Fix}^{\mathscr{A}}, x, y\right) \rightarrow \exists X\left[\mathrm{Cl}^{\mathscr{A}}(X) \wedge \neg B_{1}(X, x, y)\right],
$$

hence our assumption yields that

$$
\forall y \exists X\left[\mathrm{Cl}^{\infty}(X) \wedge \neg B_{1}(X, x, y)\right] .
$$


Applying $\left(\Sigma_{1}^{1}-\mathrm{AC}\right)$ gives us a set $Y$ such that

$$
\forall y\left[\mathrm{Cl}^{\mathscr{\infty}}\left((Y)_{y}\right) \wedge \neg B_{1}\left((Y)_{y}, x, y\right)\right] .
$$

Now we set

$$
Z:=\left\{x: \forall y\left(x \in(Y)_{y}\right)\right\},
$$

and observe that $\mathrm{Cl}^{\mathscr{A}}(Z)$ : From $\mathscr{A}(Z, x)$ we conclude that $\forall y \mathscr{A}\left((Y)_{y}, x\right)$, and so $\forall y \mathrm{Cl}^{\mathscr{A}}\left((Y)_{y}\right)$ yields $\forall y\left(x \in(Y)_{y}\right)$. Hence, by the positivity of $B_{1}$, we have

$$
\mathrm{Cl}^{\mathscr{A}}(Z) \wedge \forall y \neg B_{1}(Z, x, y) \text {. }
$$

(ii) $B(U, u)$ is of the form $\forall y B_{1}(U, u, y)$. Assume $\exists y \neg B_{1}\left(\mathrm{Fix}^{\not}, x, y\right)$. Now the I.H. yields $\exists y \exists X\left[\mathrm{Cl}^{\infty}(X) \wedge \neg B_{1}(X, x, y)\right]$, which implies $\exists X\left[\mathrm{Cl}^{\infty}(X) \wedge \neg B(X, x)\right]$.

Our claim is now obtained effortlessly.

LEMMA 3.4. For all operator forms $\mathscr{A}$ of $\mathrm{L}_{1}(\mathrm{P}, \overrightarrow{\mathrm{Q}}, \mathrm{p}), \Sigma_{1}^{1}-\mathrm{AC}_{0}$ proves:

$$
\forall x\left[x \in \operatorname{Fix}_{\vec{Y}, \vec{y}}^{\mathscr{A}} \leftrightarrow \mathscr{A}\left(\operatorname{Fix}_{\vec{Y}, \vec{y}}^{\mathscr{A}}, \vec{Y}, x, \vec{y}\right)\right] .
$$

Proof. It remains to show that $x \in \mathrm{Fix}^{\mathscr{A}}$ implies $\mathscr{A}\left(\mathrm{Fix}^{\mathscr{A}}, x\right)$. To show the contraposition, we assume that $x \notin F^{\mathscr{A}}\left(\mathrm{Fix}^{\mathscr{A}}\right)$. By Lemma 3.3 there is a $\mathscr{A}$-closed set $Z$ with $x \notin F^{\mathscr{A}}(Z)$. Since also $F^{\mathscr{A}}(Z)$ is $\mathscr{A}$-closed, $x \notin$ Fix ${ }^{\mathscr{A}}$ follows.

Summing up, we have established that for each operator form $\mathscr{A}$ of $\mathrm{L}_{1}(\mathrm{P}, \mathrm{p})$, the intersection of all $\mathscr{A}$-closed sets is a fixed point of the operator $F^{\mathscr{A}}$, provable in $\Sigma_{1}^{1}-\mathrm{AC}_{0}$. This gives rise to the following embedding:

THEOREM 3.5. If we translate an $\mathrm{L}_{\mathrm{Fix}}$ formula $B$ to $a \mathrm{~L}_{2}$ formula $B^{*}$ by substituting each fixed point constant $\mathrm{P}^{\star}$ by the $\Pi_{1}^{1}$-definable class $\mathrm{Fix}{ }^{\mathscr{}}$, the following holds:

$$
\widehat{\mathrm{ID}}_{1} \vdash B \Longrightarrow \Sigma_{1}^{1}-\mathrm{AC} \vdash B^{*} \text {. }
$$

§4. Embedding $\mid D_{1}^{*}\left\lceil\right.$ into $\Sigma_{1}^{1}-D C_{0}$. The theory $\Sigma_{1}^{1}-A C_{0}$ proves that $F i x^{\star}$ is a subclass of every $\mathscr{A}$-closed set. When we move to the sightly stronger theory $\Sigma_{1}^{1}-\mathrm{DC}_{0}$ by strengthening the choice principle $\left(\Sigma_{1}^{1}-\mathrm{AC}\right)$, we even can prove that $\mathrm{Fix}^{\mathscr{\infty}}$ is contained in every $\mathscr{A}$-closed, $\Pi_{1}^{1}$-definable class. As a consequence, we also obtain induction along the natural numbers for $\Pi_{1}^{1}$ formulas. Thus, the aforementioned embedding extends to an embedding of $\mid D_{1}^{*}\left\lceil\right.$ into $\Sigma_{1}^{1}-D C_{0}$.

Formally, the theories $\Sigma_{1}^{1}-\mathrm{DC}$ and $\Sigma_{1}^{1}-\mathrm{DC} C_{0}$ are obtained from $\Sigma_{1}^{1}-A C$ and $\Sigma_{1}^{1}-A C_{0}$ by replacing the axiom schema $\left(\Sigma_{1}^{1}-A C\right)$ by the schema $\left(\Sigma_{1}^{1}-D C\right)$ : For each $\Sigma_{1}^{1}$ formula $A(U, V)$ of $\mathrm{L}_{2}$ we have

$\left(\Sigma_{1}^{1}\right.$-DC $\quad \forall X \exists Y A(X, Y) \rightarrow \forall Q \exists Z\left[(Z)_{0}=Q \wedge \forall x A\left((Z)_{x},(Z)_{x+1}\right)\right]$.

Note, that the theory $\Sigma_{1}^{1}-D C_{0}$ proves each instance of the axiom schema $\left(\Sigma_{1}^{1}-A C\right)$.

Next, we proof within $\Sigma_{1}^{1}-D C_{0}$ that $F i x^{\mathscr{A}}$ is the least $\Pi_{1}^{1}$-definable fixed point of the operator $F^{\mathscr{N}}$.

THEOREM 4.1. For all operator forms $\mathscr{A}$ of $\mathrm{L}_{1}(\mathrm{P}, \overrightarrow{\mathrm{Q}}, \mathrm{p})$ and each $\Pi_{1}^{1}$ formula $C(u)$ of $\mathrm{L}_{2}$, the following is provable in $\Sigma_{1}^{1}-\mathrm{DC}_{0}$ :

$$
\mathrm{Cl}_{\vec{Y}, \vec{y}}^{\mathscr{A}}(\{x: C(x)\}) \rightarrow \operatorname{Fix}_{\vec{Y}, \vec{y}}^{\mathscr{A}} \subseteq\{x: C(x)\} .
$$


Before we give the proof, we consider a simpler case to illustrate the proof idea: Suppose that $\mathscr{A}$ and $\mathscr{B}$ are operator forms and that Fix ${ }^{\mathscr{B}}$ is $\mathscr{A}$-closed. We assume that there is an $x \in \mathrm{Fix}^{\mathscr{A}}$ with $x \notin \mathrm{Fix}^{\mathscr{B}}$, and argue for a contradiction. Thereto, we construct a sequence $V_{0} \supseteq V_{1} \supseteq \ldots$ of $\mathscr{B}$-closed set, such that for all $n \in \mathrm{N}$, we have $x \notin V_{n}$ and $V_{n} \supseteq F^{\mathscr{A}}\left(\bar{V}_{n+1}\right)$. Then $W:=\bigcap_{n \in \mathrm{N}} V_{n}$ is $\mathscr{A}$-closed, but $x \notin W$.

To apply this argument in the general case, we require that every $\Pi_{1}^{1}$-definable class $\{x: C(x)\}$ is primitive recursive in a fixed point.

LEMMA 4.2 (Representation Lemma). For each $\Pi_{1}^{1}$ formula $C(U, u)$ of $\mathrm{L}_{2}$ there exists an operator form $\mathscr{A}$ of $\mathrm{L}_{1}(\mathrm{P}, \mathrm{Q}, \mathrm{p})$ and a $U$-positive $\Delta_{0}^{0}$ formula $D(U, u)$ of $\mathrm{L}_{2}$, such that $\Sigma_{1}^{1}-\mathrm{AC}_{0}$ proves: For all sets $Y$, there exists a set $T$, such that

$$
\forall x\left[D\left(\text { Fix }_{T}^{\not A}, x\right) \leftrightarrow C(Y, x)\right] .
$$

Proof. As follows e.g., from results in Simpson [18], $\Sigma_{1}^{1}-\mathrm{AC}_{0}$ proves that there is a set $T$, depending on the number and set parameters occurring in $C$, such that for all $n$,

$$
(T)_{n} \text { is a tree, } \quad \text { and } \quad C(n) \leftrightarrow\left[(T)_{n} \text { is well-founded }\right] .
$$

As usual, a tree is a set of finite sequences that is closed under initial segments. Now we define an operator $F^{\curvearrowleft}$ that collects the leafs of the trees $(T)_{n}$. If the tree $(T)_{n}$ is well-founded, then the root $\left\langle\right.$ of the tree $(T)_{n}$ is an element of Fix ${ }^{\not}$, otherwise the infinite branches and therefore the root do not enter the fixed point.

Thus, we set

$$
\mathscr{A}(\mathrm{P}, \mathrm{Q}, \mathrm{p}):=\exists n\left[\mathrm{p}=\langle y, n\rangle \wedge y \in(\mathrm{Q})_{n} \wedge\left(\forall z \in(\mathrm{Q})_{n}\right)\left(z \supset y \rightarrow z \in(\mathrm{P})_{n}\right)\right],
$$

where $z \supset y$ states that $z$ is a proper extension of the sequence $y$. It is now easy to see that

$$
\forall n\left[\langle\langle\rangle, n\rangle \in \mathrm{Fix}_{T}^{\infty} \leftrightarrow C(n)\right] .
$$

Next we return to the proof of Theorem 4.1.

Proof. Assume that $\mathscr{A}$ is an operator form and $C(u)$ a $\Pi_{1}^{1}$ formula of $L_{2}$ such that $\mathrm{Cl}^{\mathscr{A}}(\{x: C(x)\})$. We aim to prove that $x \in \mathrm{Fix}^{\mathscr{A}}$ implies $C(x)$.

Lemma 4.2 provides a set $T$, an operator form $\mathscr{B}$ of $\mathrm{L}_{1}(\mathrm{P}, \mathrm{Q}, \mathrm{p})$ and a $U$-positive $\Delta_{0}^{0}$ formula $D(U, u)$ of $L_{2}$ such that

$$
\forall x\left[D\left(\mathrm{Fix}_{T}^{\mathscr{B}}, x\right) \leftrightarrow C(x)\right] .
$$

Hence our assumption reads $\mathrm{Cl}^{\mathscr{A}}\left(\left\{x: D\left(\mathrm{Fix}_{T}^{\mathscr{B}}, x\right)\right\}\right)$. We show that this implies

$$
\begin{aligned}
& \forall X \exists Z\left[F^{D}(X) \neq \mathrm{N} \wedge \mathrm{Cl}_{T}^{\mathscr{B}}(X) \rightarrow\right. \rightarrow \\
&\left.\qquad \mathrm{Cl}_{T}^{\mathscr{B}}(Z) \wedge Z \subseteq X \wedge F^{\mathscr{A}} \circ F^{D}(Z) \subseteq F^{D}(X)\right] .
\end{aligned}
$$

Fix an arbitrary $X$, such that $\mathrm{Cl}_{T}^{\mathscr{B}}(X)$, and suppose that $F^{D}(X)$ does not contain all natural numbers. If $x \notin F^{D}(X)$, then $x \notin F^{D}\left(\mathrm{Fix}_{T}^{\mathscr{B}}\right)$, so our assumption yields $x \notin F^{\mathscr{A}} \circ F^{D}\left(\mathrm{Fix}_{T}^{\mathscr{F}}\right)$, and Lemma 3.3 provides a set $Y$ that is $\mathscr{B}$-closed with respect to $T$, such that $x \notin F^{\mathscr{A}} \circ F^{D}(Y)$. If $\mathrm{Cl}_{T}^{\mathscr{B}}(X)$ and $\mathrm{Cl}_{T}^{\mathscr{P}}(Y)$ then also $\mathrm{Cl}_{T}^{\mathscr{P}}(X \cap Y)$, thus we may assume that $Y \subseteq X$. Summarizing, we obtain

$$
\forall x \exists Y\left[x \notin F^{D}(X) \rightarrow \mathrm{Cl}_{T}^{\mathscr{B}}(Y) \wedge Y \subseteq X \wedge x \notin F^{\mathscr{A}} \circ F^{D}(Y)\right] .
$$


Now $\left(\Sigma_{1}^{1}-\mathrm{AC}\right)$ gives us a set $Y$ such that for all $x \notin F^{D}(X)$

$$
\mathrm{Cl}_{T}^{\mathscr{B}}\left((Y)_{x}\right) \wedge(Y)_{x} \subseteq X \wedge x \notin F^{\mathscr{A}} \circ F^{D}\left((Y)_{x}\right) .
$$

Therefore, if we set

$$
Z:=\bigcap_{x \notin F^{D}(X)}(Y)_{x},
$$

we have $\mathrm{Cl}_{T}^{\mathscr{B}}(Z)$ and $Z \subseteq X$ and

$$
\forall x\left[x \notin F^{D}(X) \rightarrow x \notin F^{\mathscr{A}} \circ F^{D}(Z)\right],
$$

which means $F^{\mathscr{A}} \circ F^{D}(Z) \subseteq F^{D}(X)$. Thus we have shown claim (1).

Now we suppose that there is an $x \in \mathrm{Fix}^{\mathscr{A}}$ that is not an element of $x \notin F^{D}\left(\mathrm{Fix}_{T}^{\mathscr{B}}\right)$ and argue for a contradiction. Again, Lemma 3.3 provides a set $Q$ that is $\mathscr{B}$-closed with respect to $T$ and $x \notin F^{D}(Q)$. Applying $\left(\Sigma_{1}^{1}-D C\right)$ to $(1)$ gives us a set $V$ such that $(V)_{0}=Q$ and

$\forall n\left[\mathrm{Cl}_{T}^{\mathscr{B}}\left((V)_{n}\right) \rightarrow \mathrm{Cl}_{T}^{\mathscr{G}}\left((V)_{n+1}\right) \wedge(V)_{n+1} \subseteq(V)_{n} \wedge F^{\mathscr{A}} \circ F^{D}\left((V)_{n+1}\right) \subseteq F^{D}\left((V)_{n}\right)\right]$.

One easily proves by induction that

$$
\forall n\left[\mathrm{Cl}_{T}^{\mathscr{B}}\left((V)_{n}\right) \wedge(V)_{n+1} \subseteq(V)_{n} \wedge F^{\mathscr{H}} \circ F^{D}\left((V)_{n+1}\right) \subseteq F^{D}\left((V)_{n}\right)\right] .
$$

Hence, for $W:=\bigcap_{n \in N}(V)_{n}$, we have that

$$
F^{\mathscr{A}} \circ F^{D}(W) \subseteq \bigcap_{n \in \mathrm{N}} F^{D}\left((V)_{n}\right)=F^{D}\left(\bigcap_{n \in \mathrm{N}}(V)_{n}\right)=F^{D}(W) .
$$

The second but last equality follows from the fact that $D$ is positive and $\Delta_{0}^{0}$, and that $(\forall n \in \mathrm{N})\left((V)_{n+1} \subseteq(V)_{n}\right)$. So $W \subseteq Q$ and $C^{\mathscr{A}}\left(F^{D}(W)\right)$, i.e., Fix $\subseteq F^{D}(W)$. Now $x \notin F^{D}(Q)$ yields $x \notin F^{D}(W)$, thus $x \notin \mathrm{Fix}^{\mathscr{Q}}$. A contradiction!

The following corollary is an immediate consequence of Theorem 4.1. To enhance readability, we let $\operatorname{Fix}_{\vec{Y}}^{\overrightarrow{\mathscr{A}^{*}}}$ stand for $\operatorname{Fix}_{\vec{Y}}^{\mathscr{Q}_{1}}, \ldots, \mathrm{Fix}_{\vec{Y}}^{\mathscr{Q}_{n}}$.

Corollary 4.3. For all operator forms $\mathscr{A}$ and $\overrightarrow{\mathscr{A}}$ of $\mathrm{L}_{1}(\mathrm{P}, \overrightarrow{\mathrm{Q}}, \mathrm{p})$ and each $\vec{U}$ positive arithmetical formula $B(\vec{U}, \vec{u})$ of $L_{2}, \Sigma_{1}^{1}-D C_{0}$ proves:

$$
\mathrm{Cl}^{\mathscr{A}}\left(\left\{x: B\left(\mathrm{Fix}_{\vec{Y}}^{\overrightarrow{\mathscr{A}}}, x, \vec{z}\right)\right\}\right) \rightarrow \mathrm{Fix}_{\vec{Y}}^{\mathscr{A}} \subseteq\left\{x: B\left(\mathrm{Fix}_{\vec{Y}}^{\overrightarrow{\mathscr{A}}}, x, \vec{z}\right)\right\}
$$

Proof. Note that $B\left(\operatorname{Fix}_{\vec{Y}}^{\vec{F}}, x, \vec{z}\right)$ is equivalent to a $\Pi_{1}^{1}$ formula of $L_{2}$.

REMARK 4.4. We think of $U$ as coding an ordering, and set

$$
\begin{aligned}
\operatorname{Prog}(U, V) & :=\forall x[\forall y(\langle y, x\rangle \in U \rightarrow y \in V) \rightarrow x \in V], \\
\operatorname{TI}(U, V) & :=\operatorname{Prog}(U, V) \rightarrow \operatorname{Field}(U) \subseteq V, \\
\operatorname{Wo}(U) & :=\forall Y \operatorname{TI}(U, Y) .
\end{aligned}
$$

Further, we consider the operator form

$$
\mathscr{A C C}(\mathrm{P}, \mathrm{Q}, \mathrm{p}):=\forall y[\langle y, \mathrm{p}\rangle \in \mathrm{Q} \rightarrow y \in \mathrm{P}]
$$

Observe, that $\mathrm{Cl}_{U}^{A 8 B}(V)$ is the formula $\operatorname{Prog}(U, V)$ and $W_{0}(X)$ can be written as $\forall Y\left[\mathrm{Cl}_{X}^{\infty}(Y) \rightarrow\right.$ Field $\left.(X) \subseteq Y\right]$. It is immediate, that $\mathrm{Wo}(X)$ is equivalent to 
Fix $_{X}=$ Field $(X)$. Due to Theorem $4.1, \Sigma_{1}^{1}-D C_{0}$ proves for each $\Pi_{1}^{1}$ formula $C(u)$ of $L_{2}$ that

$$
\mathrm{Wo}(X) \rightarrow\left[\mathrm{Cl}_{X}^{\infty 8 g}(\{z: C(z)\}) \rightarrow \operatorname{Field}(X) \subseteq\{z: C(z)\}\right],
$$

which is normally written as

$$
\mathrm{Wo}(X) \rightarrow \operatorname{TI}(X,\{z: C(z)\}) .
$$

It is shown, e.g., in [18], that $\left(\Pi_{1}^{1}-\mathrm{TI}\right)$ is provable in $\Sigma_{1}^{1}-\mathrm{DC}_{0}$. In this sense, Corollary 4.1 is a generalization of this result.

Since $\left(\Pi_{1}^{1}-\mathrm{Tl}\right)$ implies induction along the natural numbers for all $\Pi_{1}^{1}$ formulas of $L_{2}$, the embedding given in the previous section extends to an embedding of $\mid D_{1}^{*} \uparrow$ and $I D_{1}^{*}$ into $\Sigma_{1}^{1}-D C_{0}$ and $\Sigma_{1}^{1}-D C$, respectively.

THEOREM 4.5. If we translate an $\mathrm{L}_{\mathrm{Fix}}$ formula $B$ to $a \mathrm{~L}_{2}$ formula $B^{*}$ by substituting each fixed point constant $\mathrm{P}^{\mathscr{A}}$ by the $\Pi_{1}^{1}$-definable class $\mathrm{Fix}^{\mathscr{A}}$, then the following holds:

$$
\left|\mathrm{D}_{1}^{*}\right| \vdash B \Longrightarrow \Sigma_{1}^{1}-\mathrm{DC}_{0} \vdash B^{*} \quad \text { and } \quad \mathrm{ID}_{1}^{*} \vdash B \Longrightarrow \Sigma_{1}^{1}-\mathrm{DC} \vdash B^{*} \text {. }
$$

Since $\left|\Sigma_{1}^{1}-D C_{0}\right|=\varphi \omega 0$ and $\left|\Sigma_{1}^{1}-D C\right|=\varphi \varepsilon_{0} 0$, this answers the question for a sharp upper bound of $I_{1}^{*}$ :

COROLLARY 4.6.

$$
|| D_{1}^{*}|| \leq \varphi \omega 0, \quad \text { and } \quad|| D_{1}^{*} \mid \leq \varphi \varepsilon_{0} 0 .
$$

\$5. The theories $\mid D_{\alpha}^{*}$ and $\left.I D_{\alpha}^{*}\right\rceil$. To formulate transfinite iterations of the theories $\mid D_{1}^{*}$ and $\mid D_{1}^{*} \uparrow$, we follow the lines chosen by Jäger, Kahle, Setzer and Strahm [9] and we presuppose the same ordinal-theoretic facts. Again, (OT, $\triangleleft)$ is a standard notation system based on the ternary Veblen or $\varphi$-function. As usual, we write 0 for the least element of OT with respect to the primitive recursive ordering $\triangleleft$. Ordinals are often identified with their notations. If an ordinal $\alpha$ appears within a formal argument, the closed term representing its notation is meant instead. Also, we do not distinguish between operations on ordinals and the primitive recursive analogues on their codes. By $\Phi_{0}$ we denote the least ordinal greater than 0 such that with $\alpha<\Phi_{0}$ also $\varphi 1 \alpha 0<\Phi_{0}$. We restrict ourselves to ordinals below $\Phi_{0}$ because we only bother to fix fundmental sequences for these ordinals in the subsequent well-ordering proof. However, it is straight foreward to extend the following to all ordinals below $\Phi_{1}$, the least ordinal greater than 0 which is closed under all $n$-ary $\varphi$-functions.

The language $L_{1}$ and operator forms $\mathscr{A}$ are defined as in Section 2, but this time, we extend the language $L_{1}$ by a unary relation symbol $P^{\mathscr{A}}$ for each operator form $\mathscr{A}(\mathrm{P}, \mathrm{Q}, \mathrm{p}, u)$ of $\mathrm{L}_{1}(\mathrm{P}, \mathrm{Q}, \mathrm{p})$ which contains at most the variable $u$ free, and denote this new language again by $\mathrm{L}_{\mathrm{Fix}}$. To simplify the notation, $t \in \mathrm{P}_{s}^{\mathscr{A}}$ stands for $\mathrm{P}^{\mathscr{A}}(\langle t, s\rangle)$ and $t \in \mathrm{P}_{\triangleleft s}^{\mathscr{A}}$ is to abbreviate $t=\left\langle(t)_{0},(t)_{1}\right\rangle \wedge(t)_{1} \triangleleft s \wedge t \in \mathrm{P}^{\mathscr{A}}$. For each ordinal $\alpha$ less than $\Phi_{0}$, the theory $\mathrm{ID}_{\alpha}^{*}$ comprises the axioms of PA without induction, the axioms $\mathrm{TI}_{\triangleleft}(A, \max \{\alpha, \omega\})$ for all $\mathrm{L}_{\mathrm{Fix}}$ formulas $A$ and the following fixed point axioms:

$$
(\forall a \triangleleft \alpha) \forall x\left[x \in \mathrm{P}_{a}^{\mathscr{A}} \leftrightarrow \mathscr{A}\left(\mathrm{P}_{a}^{\mathscr{A}}, \mathrm{P}_{\triangleleft a}^{\mathscr{A}}, x, a\right)\right]
$$


and

$$
\begin{aligned}
\left(\mathrm{IND}_{\mathrm{FIX}}^{+}\right) \quad(\forall a \triangleleft \alpha)\left[\mathrm{Cl}_{\mathrm{P}_{\triangleleft a}^{\mathscr{A}}, a}^{\mathscr{a}}\left(\left\{x: B\left(\mathrm{P}_{a}^{\vec{\alpha}}, \mathrm{P}_{\triangleleft a}^{\vec{A}}, x, \vec{y}\right)\right\}\right)\right. & \rightarrow \\
\mathrm{P}_{a}^{\mathscr{A}} & \left.\subseteq\left\{x: B\left(\mathrm{P}_{a}^{\vec{A}}, \mathrm{P}_{\triangleleft a}^{\vec{A}}, x, \vec{y}\right)\right\}\right],
\end{aligned}
$$

for all operator forms $\overrightarrow{\mathscr{A}}(\mathrm{P}, \mathrm{Q}, \mathrm{p}, u)$ containing at most the variable $u$ free and each $\vec{P}$-positive formula $B(\overrightarrow{\mathrm{P}}, \overrightarrow{\mathrm{Q}}, \mathrm{p}, \vec{u})$ of $\mathrm{L}_{1}(\overrightarrow{\mathrm{P}}, \overrightarrow{\mathrm{Q}}, \mathrm{p})$. In $\left|\mathrm{D}_{\alpha}^{*}\right|$, only restricted (transfinite) induction is available, i.e., instead of $\operatorname{TI}_{\triangleleft}(A, \max \{\alpha, \omega\})$, we only have

$$
(\forall a \triangleleft \alpha)\left[\operatorname{Prog}_{\triangleleft}\left(\lambda b \cdot B\left(\mathrm{P}_{a}^{\vec{A}}, \mathrm{P}_{\triangleleft a}^{\overrightarrow{\mathscr{A}}}, b, \vec{y}\right)\right) \rightarrow(\forall c \triangleleft \max \{\alpha, \omega\}) B\left(\mathrm{P}_{a}^{\vec{A}}, \mathrm{P}_{\triangleleft a}^{\vec{A}}, c, \vec{y}\right)\right]
$$

for each $\vec{P}$-positive formula $B(\vec{P}, \vec{Q}, \mathrm{p}, \vec{u})$ of $\mathrm{L}_{1}(\overrightarrow{\mathrm{P}}, \overrightarrow{\mathrm{Q}}, \mathrm{p})$. As usual, for a formula $A(u)$ of $\mathrm{L}_{\text {Fix }}, \operatorname{Prog}_{\prec}(\lambda x . A(x))$ abbreviates $\forall u(\forall v \prec u)(A(v) \rightarrow A(u))$. Note that the axioms concerning transfinite induction imply also induction along the natural numbers for the corresponding class of formulas. Again, $\widehat{I D}_{\alpha}$ is $I_{\alpha}^{*}$ without $\left(I N D_{\mathrm{FIX}}^{+}\right)$.

§6. Wellordering proofs for $\mid D_{\alpha}^{*}$ and $I D_{\alpha}^{*} \uparrow$. To demonstrate the power of the axiom $\left(\mid N_{\mathrm{FIX}}^{+}\right)$, we give wellordering proofs for the theories $\mid \mathrm{D}_{\alpha}^{*}$ and $\left.\mid \mathrm{D}_{\alpha}^{*}\right\rceil$. The proof idea is the same as in [9], where the wellordering proof of $\widehat{I D}_{\alpha}$ is carried out in the transfinitely iterated theory of self-reflecting truth $\mathrm{SRT}_{\alpha}$. However, things are easier in the present context and the wellordering proof is performed in $I D_{\alpha}^{*}$ itself. As Corollary 4.6 suggests, we obtain that also $\mathrm{ID}_{\alpha}^{*}$ and $\widehat{\mathrm{ID}}_{\alpha}$ prove the same ordinals.

For the wellordering proof, we fix fundamental sequences for the ordinals below $\Phi_{0}$. A fundamental sequence for $\alpha$ is a primitive recursive, increasing sequence $\alpha[n]$ on the corresponding notations such that for each $\beta<\alpha<\Phi_{0}$ there is an $n$ with $\beta \leq \alpha[n]$. We set $(\alpha+1)[n]:=\alpha$ for all $n \in N$, and if $\omega^{\alpha_{k}}+\cdots+\omega^{\alpha_{1}}$ is the Cantor normal form of $\lambda$ and $\lambda\left\langle\omega^{\lambda}\right.$, then $\lambda[n]:=\omega^{\alpha_{k}}+\cdots+\omega^{\alpha_{2}}+\omega^{\alpha_{1}}[n]$. The remaining cases where $\lambda<\Phi_{0}$ is of the form $\varphi \alpha \beta \gamma$ for $\alpha \in\{0,1\}$ and $\beta, \gamma<\lambda$ are given next: $\Gamma_{0}[0]:=0$ and $\Gamma_{0}[n+1]:=\varphi\left(\Gamma_{0}[n]\right) 0, \varphi 00(\gamma+1)[n]=\omega^{\gamma+1}[n]:=\omega^{\gamma} \cdot n$, and

(i) $\varphi \alpha(\beta+1) 0[0]=0$ and $\varphi \alpha(\beta+1) 0[n+1]=\varphi \alpha \beta(\varphi \alpha(\beta+1) 0[n])$.

(ii) For a limit $\lambda: \varphi \alpha \beta \lambda[n]=\varphi \alpha \beta(\lambda[n])$ and $\varphi \alpha \lambda 0[n]=\varphi \alpha(\lambda[n]) 0$.

(iii) $\varphi \alpha \beta(\gamma+1)[0]=\varphi \alpha \beta \gamma+1$, and

$$
\begin{aligned}
\varphi \alpha \beta(\gamma+1)[n+1] & =\varphi \alpha(\beta[n])(\varphi \alpha \beta(\gamma+1)[n]), \text { if } \beta>0, \\
& =\varphi 0(\varphi 10(\gamma+1)[n]) 0, \text { if } \beta=0 \text { and } \alpha=1 .
\end{aligned}
$$

In the course of the wellordering proof, we let $a, b, c, d, e$ range over the elements of OT and use $l$ to denote limit notations. We start with the cases $I D_{1}^{*}$ and $I D_{1}^{*} \mid$.

Let $\mathscr{C}:=(\forall z \triangleleft x)(z \in \mathrm{P})$ and denote the corresponding fixed point constant $\mathrm{P}^{\mathscr{A}}$ by ACC. By means of the axiom (IND $\mathrm{FIX}^{+}$) one immediately proves in $\mathrm{ID}_{1}^{*} \uparrow$ that $a, b \in \mathrm{ACC}$ implies ACC $\subseteq\{c: a+c \in \mathrm{ACC}\}$ and $\mathrm{ACC} \subseteq\{c: a \cdot c \in \mathrm{ACC}\}$, hence $a, b \in \mathrm{ACC}$ yields $a+b \in \mathrm{ACC}$ and $a \cdot b \in \mathrm{ACC}$.

LEMMA 6.1. For each ordinal $k<\omega$, and each ordinal $\kappa<\varepsilon_{0}$, the following holds:

$$
\mathrm{ID}_{1}^{*} \vdash \vdash \operatorname{Prog}_{\triangleleft}(\lambda a . \varphi k a \in \mathrm{ACC}) \quad \text { and } \mathrm{ID}_{1}^{*} \vdash \operatorname{Prog}_{\triangleleft}(\lambda a . \varphi \kappa a \in \mathrm{ACC}) \text {. }
$$

Proof. Note that $\operatorname{Prog}_{\triangleleft}(\lambda x . A(x))$ is another way of writing $\mathrm{Cl}^{188}(\{x: A(x)\})$. We prove the first claim by (meta-) induction on $k$. For $k=0$, it is to show that 
if $\omega^{b} \in$ ACC holds for all $b \triangleleft a$, then also $\omega^{a} \in$ ACC. If $a$ is a limit notation, this follows from $\operatorname{Prog}_{\triangleleft}(\lambda b . b \in \mathrm{ACC})$ and the continuity of the function $\lambda \xi . \omega^{\xi}$. If $a$ is of the form $b+1$, then we use restricted induction to show that $\forall n\left(\omega^{\beta} \cdot n \in A C C\right)$, thus $\operatorname{Prog}_{\triangleleft}(\lambda b . b \in \mathrm{ACC})$ yields $\omega^{\beta+1} \in \mathrm{ACC}$.

For the induction step, we assume that $\varphi(k+1) b \in$ ACC for all $b \triangleleft a$. Now the I.H. allows to prove by restricted induction that $\forall n(\varphi(k+1) a[n] \in A C C)$. Thus, also $\varphi(k+1) a \in$ ACC.

For the second claim, observe that in $I D_{1}^{*}$ transfinite induction along ordinals $\kappa<\varepsilon_{0}$ is available for all formulas of $L_{F i x}$. Instead of meta-induction, transfinite induction within $I D_{1}^{*}$ is used. If $\lambda$ is a limit ordinal, the induction step is performed by showing $\forall n(\varphi \lambda a[n] \in \mathrm{ACC})$.

The axiom $\left(\mathrm{IND}_{\mathrm{FIX}}^{+}\right)$implies $\operatorname{Prog}_{\triangleleft}(\mathrm{U}) \rightarrow A C C \subseteq U$. Since the previous lemma yields $\left|D_{1}^{*}\right| \vdash \varphi k 0 \in A C C$ and $I D_{1}^{*} \vdash \varphi \kappa 0 \in A C C$ for $k<\omega$ and $\kappa<\varepsilon_{0}$, Theorem 4.6 gives rise to the following corollary.

COROLlaRY 6.2 .

$$
\mid I D_{1}^{*} \|=\varphi \omega 0, \quad \text { and } \quad\left|I D_{1}^{*}\right|=\varphi \varepsilon_{0} 0 .
$$

Next we consider $I D_{\alpha}^{*}\left\lceil\right.$ and $I D_{\alpha}^{*}$. By mentioning $I D_{\alpha}^{*}\left\lceil\right.$ or $I D_{\alpha}^{*}$, we implicitly imply $\alpha<\Phi_{0}$. This time, let $\mathscr{A C}:=(\forall z \triangleleft p)\left(z \in \mathrm{P}_{a}\right)$ and denote the corresponding relation symbol $\mathrm{P}^{\mathscr{A}}$ by $\mathrm{ACC}$. In the sequel we write $c \in \mathrm{ACC}^{a}$ for $(\forall b \triangleleft a)(c \in$ $\left.A C C_{b}\right)$. Note that this reads $(\forall b \triangleleft a)\left(\langle c, b\rangle \in A C C_{\triangleleft a}\right)$. Therefore $\mid D_{\alpha}^{*}\lceil$ proves for each formula $B(\mathrm{P}, \mathrm{p})$ of $\mathrm{L}_{1}(\mathrm{P}, \mathrm{p})$, that $a \triangleleft \alpha$ and $\mathrm{Prog}_{\triangleleft}\left(\lambda x \cdot B\left(\mathrm{ACC}^{a}, x\right)\right)$ imply $\mathrm{ACC}_{a} \subseteq\left\{x: B\left(\mathrm{ACC}^{a}, x\right)\right\}$. Further, $a \triangleleft \alpha$ implies the progressivity of $\mathrm{ACC}^{a}$ and $b \triangleleft a \triangleleft \alpha$ implies $\mathrm{ACC}_{a} \subseteq \mathrm{ACC}_{b}$. Since $\mathrm{Prog}_{\triangleleft}(\mathrm{U})$ implies $\mathrm{ACC}_{0} \subseteq \mathrm{U}$, proving an ordinal $\beta$ in $\mathrm{ID}_{\alpha}^{*} \mid$ breaks down to show $\beta \in \mathrm{ACC}_{0}$.

LEMMA 6.3. Let $A(a, b):=\forall c\left(c \in \mathrm{ACC}_{a} \rightarrow \varphi b c \in \mathrm{ACC}_{a}\right)$. Then it is provable in $\mathrm{ID}_{\alpha}^{*}\left\lceil\right.$ that $a \triangleleft \alpha \rightarrow \operatorname{Prog}_{\triangleleft}(\lambda b . A(a, b))$.

Proof. Let $a \triangleleft \alpha$. We assume $\left(\forall b^{\prime} \triangleleft b\right) A\left(a, b^{\prime}\right)$ and show $A(a, b)$.

(IND年|X $)$ tells us that $A(a, b)$ follows from $\operatorname{Prog}_{\triangleleft}\left(\lambda c . \varphi b c \in \mathrm{ACC}_{a}\right)$, which in turn follows from the assumption $\left(\forall b^{\prime} \triangleleft b\right) A\left(a, b^{\prime}\right)$ : Given $\varphi b c^{\prime} \in \mathrm{ACC}_{a}$ for all $c^{\prime} \triangleleft c$, restricted induction yields that $\forall n\left(\varphi b c[n] \in \mathrm{ACC}_{a}\right)$, thus $\varphi b c \in \mathrm{ACC}_{a}$.

COROLLARY 6.4. For all limit notations $l \unlhd \alpha, \mathrm{ID}_{\alpha}^{*}\lceil$ proves:

$$
d \in \mathrm{ACC}^{l} \rightarrow \varphi d 0 \in \mathrm{ACC}^{l} .
$$

PRoof. Pick an arbitrary $a \triangleleft l$. So $d \in \mathrm{ACC}^{l}$ implies $d \in \mathrm{ACC}_{a+1}$. Now (IND $\mathrm{FIX}^{+}$) and $\operatorname{Prog}_{\triangleleft}\left(\lambda b . \forall c\left(c \in \mathrm{ACC}_{a} \rightarrow \varphi b c \in \mathrm{ACC}_{a}\right)\right)$ yield $\varphi d 0 \in \mathrm{ACC}_{a}$.

Corollary 6.5. For all limit notations $l \triangleleft \alpha, \mid D_{\alpha}^{*}\lceil$ proves:

$$
\operatorname{Prog}_{\triangleleft}\left(\lambda c . \varphi 10 c \in \mathrm{ACC}^{l}\right) \text {. }
$$

Proof. Assume that $l \triangleleft \alpha$ and that $\varphi 10 d \in \mathrm{ACC}^{l}$ for all $d \triangleleft c$. Restricted induction and the previous corollary imply $\forall n\left(\varphi 10 c[n] \in \mathrm{ACC}^{l}\right)$. Thus $\varphi 10 c \in \mathrm{ACC}^{l}$.

COROLLARY 6.6. For all limits $l \unlhd \alpha, \mathrm{ID}_{\alpha}^{*}$ proves:

$$
\operatorname{Prog}_{\triangleleft}\left(\lambda c . \varphi 10 c \in \mathrm{ACC}^{l}\right) \text {. }
$$


Proof. In the case $l=\alpha$, full induction is needed to show $\forall n\left(\varphi 10 c[n] \in \mathrm{ACC}^{l}\right)$.

The following lemma corresponds to the Main Lemma in [9]. Again, the proof is simpler in the present context.

LEMMA 6.7. Let

$$
A(a, b):=\forall d, c\left(d+\omega^{1+b} \unlhd a \wedge c \in \mathrm{ACC}_{d+\omega^{1+b}} \rightarrow \varphi 1 b c \in \mathrm{ACC}^{d+\omega^{1+b}}\right) .
$$

Then it is provable in $\mathrm{ID}_{\alpha}^{*} \mid$ that $a \triangleleft \alpha \rightarrow \operatorname{Prog}_{\triangleleft}(\lambda b . A(a, b))$.

Proof. Assume $a \triangleleft \alpha$ and that $A\left(a, b^{\prime}\right)$ holds for all $b^{\prime} \triangleleft b$. We aim for $A(a, b)$. So suppose $d+\omega^{1+b} \unlhd a$. Now $c \in \mathrm{ACC}_{d+\omega^{1+b}} \rightarrow \varphi 1 b c \in \mathrm{ACC}^{d+\omega^{1+b}}$ follows, if we can establish

$$
\operatorname{Prog}_{\triangleleft}\left(\lambda c . \varphi 1 b c \in \mathrm{ACC}^{d+\omega^{1+b}}\right) .
$$

Thereto we further suppose that $\varphi \mathrm{l} b c^{\prime} \in \mathrm{ACC}^{d+\omega^{1+b}}$ for all $c^{\prime} \triangleleft c$, and use restricted induction to show $\forall n\left(\varphi 1 b c[n] \in A C C^{d+\omega^{1+b}}\right)$. We only consider the case where $b$ is not 0 and $c$ a successor: $\varphi 1 b c[0] \in \mathrm{ACC}^{d+\omega^{1+b}}$ follows immediately from our further supposition, and the induction step can be performed because we have for all $m \in N$,

$$
\forall e\left(e \in \mathrm{ACC}^{d+\omega^{1+b}} \rightarrow \varphi 1(b[m]) e \in \mathrm{ACC}^{d+\omega^{1+b}}\right) .
$$

To see that (2) holds, fix an $m \in \mathrm{N}$ and suppose that $e \in \mathrm{ACC}^{d+\omega^{1+b}}$. We argue that $\varphi 1(b[m]) e \in \mathrm{ACC}_{d+\omega^{1+b}[k]}$ for all $k \in \mathrm{N}$ : So we fix an arbitrary $k \in \mathrm{N}$, and

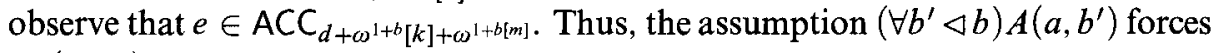
$\varphi 1(b[m]) e \in \mathrm{ACC}_{d+\omega^{1+b}[k]}$.

The other cases are shown similarly or are easy. If $b=0$, (1) becomes Corollary 6.5.

From the above proof we immediately extract the following corollaries:

COROLLARY 6.8. For all notations $b$ and all $d$ with $d+\omega^{1+b} \triangleleft \alpha, \mathrm{ID}_{\alpha}^{*} \mid$ proves:

$$
\operatorname{Prog}_{\triangleleft}\left(\lambda c . \varphi 1 b c \in \mathrm{ACC}^{d+\omega^{1+b}}\right) .
$$

CoRollaRY 6.9. For all notations $b$ and all $d$ with $d+\omega^{1+b} \unlhd \alpha, \mathrm{ID}_{\alpha}^{*}$ proves:

$$
\operatorname{Prog}_{\triangleleft}\left(\lambda c . \varphi 1 b c \in \mathrm{ACC}^{d+\omega^{1+b}}\right) .
$$

Proof. Let $A(a, b)$ be as defined in Lemma 6.7. By transfinite induction we obtain $A(a, b)$ for all $a \triangleleft \alpha$ and $b \unlhd \alpha$. Using full induction, the claim is shown as in the proof of Lemma 6.7.

In order to speak about lower and upper bounds of $\mid D_{\alpha}^{*}$ and $\left.\mid D_{\alpha}^{*}\right\rceil$, we define for all $\alpha, \beta<\Phi_{0}$ a function $\sigma(\alpha, \beta)$.

DEFINITION 6.10. Let $\alpha=\omega^{1+\alpha_{n}}+\omega^{1+\alpha_{n-1}}+\cdots+\omega^{1+\alpha_{1}}+m$, where $\alpha_{n} \geq \cdots \geq \alpha_{1}$ and $m<\omega$, be an ordinal below $\Phi_{0}$ in Cantor normal form. We set for all ordinals $\beta<\Phi_{0}$ :

$$
\sigma(\alpha, \beta):=\varphi 1 \alpha_{n}\left(\varphi 1 \alpha_{n-1}\left(\ldots\left(\varphi 1 \alpha_{1} \beta\right) \ldots\right), \text { if } \alpha \geq \omega \text { and } \sigma(m, \beta):=\beta .\right.
$$

Moreover, $(\alpha \mid 0):=\varepsilon(\alpha)$, i.e., the least fixed point of the function $\lambda \xi . \omega^{\xi}$ bigger than $\alpha$, and $(\alpha \mid i+1):=\varphi(\alpha \mid i) 0$. 
$\left(\alpha\lceil 0)\right.$ is the least limit ordinal $\lambda>0$ such that $\varphi 1 \alpha_{1} \lambda>\alpha$, and $(\alpha\lceil 1)$ is the least upper bound of $\{\varphi k(\alpha+1): k<\omega\}$. Eventually, $(\alpha \mid i+2):=\varphi(\alpha\lceil i+1) 0$.

Towards further simplifications, we write in the sequel $\sigma(\alpha)$ for $\sigma(\alpha,(\alpha \mid m))$ and $\sigma\lceil(\alpha)$ for $\sigma(\alpha,(\alpha\lceil m))$.

Note, that $\alpha\lceil 0$ is of the form $\beta+\omega$, where $\beta$ is a limit or zero, and if $\beta>0$, then $\alpha \geq \varphi 1 \alpha_{1} \beta$ : Let $\beta_{0}:=\min \left\{\beta: \varphi 1 \alpha_{1} \beta \geq \alpha\right\}$. Now if $\beta_{0}$ is zero or a successor, the claim is immediate, and if $\beta_{0}$ is a limit, then the continuity of the function $\lambda \xi . \varphi 1 \alpha_{1} \xi$ yields $\varphi 1 \alpha_{1} \beta_{0}=\alpha$, thus $\alpha \mid 0=\beta_{0}+\omega$.

LEMMA 6.11. Let $\lambda=\omega^{1+\alpha_{n}}+\omega^{1+\alpha_{n-1}}+\cdots+\omega^{1+\alpha_{1}}<\Phi_{0}$, where $\alpha_{n} \geq \cdots \geq \alpha_{1}$, and assume that $\lambda\left\lceil 0=\beta+\omega\right.$. Then, for each $n \in \mathrm{N}, \mathrm{ID}_{\lambda}^{*}\lceil$ proves the following:

$$
\varphi 1 \alpha_{1}(\beta+n) \in \mathrm{ACC}^{\lambda} \text {. }
$$

Proof. We choose $\delta$ such that $\lambda=\delta+\omega^{1+\alpha_{1}}$ and prove the claim by metainduction on $n$. We start with the case $n=0$ : If $\beta>0$, then $\varphi 1 \alpha_{1} \beta \leq \lambda$ and the claim trivially follows by restricted transfinite induction up to $\lambda$. If $\beta=0$, then the claim follows similar to the induction step, which we prove below. Thereby, we distinguish whether $\alpha_{1}$ is zero, a successor or a limit. Exemplarily, we show the case $\alpha_{1}=\alpha_{1}^{\prime}+1$ : To establish $\varphi 1 \alpha_{1}(\beta+n+1) \in \mathrm{ACC}^{\delta+\omega^{1+\alpha_{1}}}$, we show that for all $k \in \mathrm{N}, \varphi 1 \alpha_{1}(\beta+n+1)[k] \in \mathrm{ACC}^{\delta+\omega^{1+\alpha_{1}^{\prime} \cdot k}}$ by proving, using restricted induction on $m$, that for each $k \in \mathrm{N}$,

$$
(\forall m \leq k)\left[\varphi 1 \alpha_{1}(\beta+n+1)[m] \in \mathrm{ACC}_{\delta+\omega^{1+\alpha_{1}^{\prime} \cdot(2 k-m)}}\right]:
$$

If $m<k$, then the I.H. yields $\varphi 1 \alpha_{1}(\beta+n+1)[m] \in \mathrm{ACC}_{\delta+\omega^{1+\alpha_{1}^{\prime}} \cdot(2 k-m)}$ and Corollary 6.8 tells us

$$
\operatorname{Prog}_{\triangleleft}\left(\lambda c . \varphi 1 \alpha_{1}^{\prime} c \in \mathrm{ACC}^{\delta+\omega^{1+\alpha_{1}^{\prime}} \cdot(2 k-m)}\right) .
$$

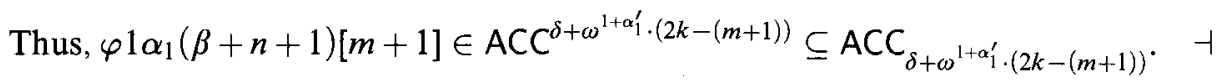

We conclude this section by presenting the lower bounds:

THEOREM 6.12. For all $0<\alpha<\Phi_{0}$ we have:

$$
\left.|| \mathrm{D}_{\alpha}^{*} \mid \geq \sigma(\alpha) \quad \text { and } \quad \mid \mathrm{ID}_{\alpha}^{*}\right\rceil \geq \sigma \uparrow(\alpha) .
$$

Proof. Assume that $\alpha=\omega^{1+\alpha_{n}}+\cdots+\omega^{1+\alpha_{1}}+m$ for ordinals $\alpha_{n} \geq \cdots \geq \alpha_{1}$ and $m<\omega$, and set $\delta_{k}:=\omega^{1+\alpha_{n}}+\cdots+\omega^{1+\alpha_{k}}$ for $k \leq n$, and

$\sigma_{k}:=\varphi 1 \alpha_{k}\left(\ldots \varphi 1 \alpha_{1}(\alpha\lceil m)) \ldots\right)$. By meta-induction on $k$ we now show that for all $\beta<\sigma_{k}$, the theory $\mathrm{ID}_{\alpha}^{*} \mid$ proves $\beta \in \mathrm{ACC}^{\delta_{k}}$ :

We first consider the case $k=1$. If $m=0$, then $\delta_{1}=\alpha$ and $\sigma_{1}=\varphi 1 \alpha_{1}(\alpha\lceil 0)$. Hence the claim follows by Lemma 6.11. If $m=m^{\prime}+1$, then there exists for each $\beta<\sigma_{1}=\varphi 1 \alpha_{1}(\alpha \mid 1)$ a $k<\omega$ and ordinals $\xi_{1}, \ldots, \xi_{m}$ such that $\xi_{1}=\varphi k(\alpha+1)$ and $\xi_{i+i}=\varphi \xi_{i} 0$ and $\beta<\varphi 1 \alpha_{1} \xi_{m}$. It follows from the proof of Lemma 6.1 that $\mathrm{ID}_{\alpha}^{*} \mid$ proves the progressivity of $\lambda a . \varphi k a \in \mathrm{ACC}^{\delta_{1}+m^{\prime}}$, thus $\xi_{1} \in \mathrm{ACC}_{\delta_{1}+m^{\prime}}$. Applying $m^{\prime}$-times Lemma 6.3 and $\left(\mathrm{IND}_{\mathrm{FIX}}^{+}\right)$yields $\xi_{m} \in \mathrm{ACC}_{\delta_{1}}$. Now Lemma 6.7 yields $\varphi 1 \alpha_{1} \xi_{m} \in \mathrm{ACC}^{\delta_{1}}$.

The induction step from $k$ to $k+1$ follows with Corollary 6.8 .

The case ID $\mathrm{ID}_{\alpha}^{*}$ is treated similarly. If $m=0$, we use that for all formulas $A(u)$ of $\mathrm{L}_{\mathrm{Fix}}, \mathrm{ID}_{\alpha}^{*}$ proves $\operatorname{Prog}_{\triangleleft}(\lambda a . A(a)) \rightarrow A(\beta)$, for all $\beta<\varepsilon(\alpha)$.

That these bounds are sharp is established in the next section. 
\$7. Upper bounds for $I D_{\alpha}^{*}$ and $I D_{\alpha}^{*}\lceil$. The aim of this section is to determine the upper bounds of the $I^{*}$-theories $I_{\alpha}^{*}$ and $I D_{\alpha}^{*} \uparrow$. In a first step, we introduce for each ordinal $\alpha<\Phi_{0}$ a theory $\mathrm{M}_{\alpha}$ which formalizes an $\alpha$-hierarchy of models of $\Sigma_{1}^{1}-A C$. Then upper bounds of the theories $\left(M_{\alpha}+A C A\right)_{0},\left(M_{\alpha}+\Sigma_{1}^{1}-D C\right)_{0}$ and $M_{\lambda} \Gamma$, the so called $M$-theories, are identified by reducing them to semi-formal systems $\mathrm{E}_{\alpha}^{0}$, presented and analyzed by Rüede in [15]. Next we extend each M-theory to a corresponding $\mathrm{M}^{\dagger}$-theory by adding the axiom $\neg T l_{\triangleleft}(U, \xi)$, where $\xi$ is the previously determined upper bound of the M-theory and argue that $\xi$ is still an upper bound of the corresponding $\mathrm{M}^{\dagger}$-theory. Finally, we give embeddings of the ID*-theories into the $\mathrm{M}^{\dagger}$-theories, namely $\mathrm{ID}_{\alpha}^{*}$ into $\left(\mathrm{M}_{\alpha}+\mathrm{ACA}\right)_{0}^{\dagger}, \mathrm{ID}_{\alpha+1}^{*}\left\lceil\right.$ into $\left(\mathrm{M}_{\alpha}+\Sigma_{1}^{1}-\mathrm{DC}\right)_{0}^{\dagger}$ and $I D_{\lambda}^{*}\left\lceil\right.$ into $\left(M_{\lambda} \uparrow\right)^{\dagger}$ if $\lambda$ is a limit.

7.1. Upper bounds for $\left(M_{\alpha}+A C A\right)_{0},\left(M_{\alpha}+\Sigma_{1}^{1}-D C\right)_{0}$ and $M_{\lambda} \uparrow$. For each $\alpha<\Phi_{0}$, the theory $M_{\alpha}$ is formulated in the language $L_{2}(D)$ which extends $L_{2}$ by the unary relation symbol D. Formulas of $L_{2}(D)$ that do not contain bound set variables are called elementary. To simplify the notation, we write $t \in \mathrm{D}$ for $\mathrm{D}(t), t \in \mathrm{D}_{s}$ for $\langle t, s\rangle \in \mathrm{D}$ and $X \dot{\in} \mathrm{D}_{s}$ is to abbreviate the formula $\exists x\left(X=\left(\mathrm{D}_{s}\right)_{x}\right)$, where again, $X=\left(D_{s}\right)_{t}$ is short for $\forall x\left(x \in X \leftrightarrow\langle x, t\rangle \in D_{s}\right)$. The expression $t \in X_{\triangleleft a}$ stands for $t=\left\langle(t)_{0},(t)_{1}\right\rangle \wedge(t)_{1} \triangleleft a \wedge t \in X$ and $t \in D_{\triangleleft a}$ is defined accordingly. $X \dot{\in} \mathrm{D}_{\triangleleft a}$ is read as $(\exists b \triangleleft a)\left(X \dot{\in} \mathrm{D}_{b}\right)$. The relativization $A^{\mathrm{D}_{a}}$ of an $\mathrm{L}_{2}(\mathrm{D})$ formula to $\mathrm{D}_{a}$ is $A$ for an elementary $A,(\forall X A(X))^{\mathrm{D}_{a}}:=\forall x A^{\mathrm{D}_{a}}\left(\left(\mathrm{D}_{a}\right)_{x}\right)$ and $(\exists X A(X))^{\mathrm{D}_{a}}$ is $\exists x A^{\mathrm{D}_{a}}\left(\left(\mathrm{D}_{a}\right)_{x}\right)$. Relativizations to $\mathrm{D}_{\triangleleft a}$ are defined analogously. Observe that if $A$ is an $\mathrm{L}_{2}(\mathrm{D})$ formula without free set variables, then $A^{\mathrm{D}_{a}}$ is a formula of $\mathrm{L}_{1}(\mathrm{D})$. Finally, $\mathrm{Ax}_{\Sigma_{1}^{1}-A C}$ denotes the finite axiomatization of $\Sigma_{1}^{1}-A C$ given in [14], namely the conjunction of the formulas listed below:

(i) $\forall X, Y \exists Z(Z=X \oplus Y)$,

(ii) $\forall e, z, Z \exists Y \forall x\left[x \in Y \leftrightarrow \pi_{1}^{0}(Z, e, x, z)\right]$,

(iii) $\forall e, z, Z\left[\forall x \exists X \pi_{2}^{0}(X, Z, e, x, z) \rightarrow \exists Y \forall x \pi_{2}^{0}\left((Y)_{x}, Z, e, x, z\right)\right]$,

where $\pi_{k}^{0}$ is a universal $\Pi_{k}^{0}$ formula of $\mathrm{L}_{2}$ of the appropriate ariety and $X \oplus Y$ denotes the set $\{\langle x, 0\rangle: x \in X\} \cup\{\langle x, 1\rangle: x \in Y\}$.

The idea is that $D$ constitutes an $\alpha$-hierarchy of models of $\Sigma_{1}^{1}-A C$, i.e., for all ordinal notations $a \triangleleft \alpha$, we have that $\mathrm{D}_{a}$ is a model of $\Sigma_{1}^{1}-\mathrm{AC}$ and $\mathrm{D}_{\triangleleft a} \dot{\in} \mathrm{D}_{a}$. Note however, that $\left[\forall x \exists X A(X, x) \rightarrow \exists Y \forall x A\left((Y)_{x}, x\right)\right]^{\mathrm{D}_{a}}$ holds only for $\Sigma_{1}^{1}$ formulas of $\mathrm{L}_{2}$, not for $\Sigma_{1}^{1}$ formulas of $\mathrm{L}_{2}(\mathrm{D})$.

In order to have partial cut elimination at hand, we formulate the $\mathrm{M}$-theories in a Tait-style calculus that extends the classical Tait-calculus (cf. [17]) by the nonlogical axioms and rules of the $\mathrm{M}$-theories. We let $\Gamma, \Delta, \ldots$ range over finite sets of $\mathrm{L}_{2}$ (D) formulas and write $\Gamma, A$ for the union of $\Gamma$ and $\{A\}$. For each $\alpha<\Phi_{0}$, the theory $\mathrm{M}_{\alpha}$ consist of the following axioms and rules:

Basic axioms. For all finite sets $\Gamma$ of $L_{2}$ (D) formulas, all elementary formulas $A$ of $L_{2}$ (D) and all arithmetical formulas $B$ of $L_{2}$ which are axioms for the primitive recursive functions and relations:

$$
\Gamma, A, \neg A \quad \text { and } \quad \Gamma, B .
$$

Propositional and quantifier rules. These include the usual Tait-style inference rules for the propositional connectives as well as number and set quantifiers. 
D-axioms. For all finite sets $\Gamma$ of $L_{2}(D)$ formulas:

$\Gamma, a \triangleleft \alpha \rightarrow \exists X\left[X=\mathrm{D}_{a}\right], \quad \Gamma, a \triangleleft \alpha \rightarrow\left(\mathrm{Ax}_{\Sigma_{1}^{l}-\mathrm{AC}}\right)^{\mathrm{D}_{a}}$ and $\Gamma, a \triangleleft \alpha \rightarrow \mathrm{D}_{\triangleleft a} \dot{\in} \mathrm{D}_{a}$ :

Transfinite induction. For all finite sets $\Gamma$ of $L_{2}(D)$ formulas:

$$
\Gamma, \operatorname{TI}_{\triangleleft}(U, \max \{\alpha, \omega\}) \text {. }
$$

Cut rules. For all finite sets $\Gamma$ of $\mathrm{L}_{2}$ (D) formulas and all $\mathrm{L}_{2}(\mathrm{D})$ formulas $A$ :

$$
\frac{\Gamma, A \quad \Gamma, \neg A}{\Gamma}
$$

The formulas $A$ and $\neg A$ are the cut formulas of this cut.

Note that the D-axioms imply that $U, V \dot{\in} D_{0}$. For limit ordinals $\lambda<\Phi_{0}$, the theory $M_{\lambda} \Gamma$ is obtained by replacing the axioms for transfinite induction by the following restricted version:

Restricted transfinite induction. For all finite sets $\Gamma$ of $L_{2}(D)$ formulas:

$$
\Gamma, a \triangleleft \lambda \rightarrow\left(\forall X \dot{\in} \mathrm{D}_{a}\right) \mathrm{TI}_{\triangleleft}(X, \lambda) \text {. }
$$

The theory $\left(M_{\alpha}+A C A\right)_{0}$ extends $M_{\alpha}$ by axioms for arithmetical comprehension and $\left(M_{\alpha}+\Sigma_{1}-D C\right)_{0}$ extends $\left(M_{\alpha}+A C A\right)_{0}$ by rules that imply all instances of dependent choice for $\Sigma_{1}^{l}$ formulas of $L_{2}$ :

Arithmetical comprehension. For all finite sets $\Gamma$ of $L_{2}(D)$ formulas and all arithmetical $\mathrm{L}_{2}$ formulas $A$ :

$$
\Gamma, \exists X[\forall x(x \in X \leftrightarrow A(x))] .
$$

Dependent choice. For all finite sets $\Gamma$ of $L_{2}(D)$ formulas and all arithmetical $L_{2}$ formulas $A$ :

$$
\frac{\Gamma, \forall X \exists Y A(X, Y)}{\Gamma, \exists Z\left[(Z)_{0}=W \wedge \forall x A\left((Z)_{x},(Z)_{x+1}\right)\right.} .
$$

The formulas mentioned beside $\Gamma$ in an axiom or the conclusion of a rule are called main formulas. Note that due to the axiom about transfinite induction, induction along the natural numbers for sets is available in $\left(M_{\alpha}+A C A\right)_{0}$ and $\left(M_{\alpha}+\Sigma_{1}^{1}-D C\right)_{0}$.

To apply the machinery developed by Rüede in [15], we aim to embed our $M$ theories into a semi-formal systems $E_{\alpha}^{0}$, that we introduce later. In a first step, we eliminate the comprehension and dependent choice part of $\left(M_{\alpha}+\Sigma_{1}^{1}-D C\right)_{0}$ and the comprehension part of $\left(M_{\alpha}+A C A\right)_{0}$. For that purpose we introduce for each $\alpha<\Phi_{0}$ a semi-formal system $\mathrm{RA}_{\alpha}$, which is essentially an extension of RA* of Schütte [16] by the D-axioms for $M_{\alpha}$.

Also the system $R A_{\alpha}$ is formulated in a Tait-style calculus. The language $L_{R A_{\alpha}}$ of $\mathrm{RA}_{\alpha}$ is the language $\mathrm{L}_{2}(\mathrm{D})$, where the set variables $X, Y, Z, \ldots$ are replaced by set variables $X^{\beta}, Y^{\beta}, Z^{\beta}, \ldots$ for each ordinal $\beta<\alpha$. In $\mathrm{RA}_{\alpha}$ we have set terms, which we define inductively together with the formulas of $L_{R A_{\alpha}}$ :

(i) Each set variable $X^{\beta}$ is a set term.

(ii) If $A(u)$ is a formula of $L_{R A_{\alpha}}$, then $\{x: A(x)\}$ is a set term.

(iii) $[\sim] \mathrm{D}(t),[\sim] \mathrm{U}(t),[\sim] \mathrm{V}(t)$ and $[\sim] R(\vec{t})$ are formulas of $\mathrm{L}_{R A_{\alpha}}$, where $R$ is a primitive recursive relation symbol.

(iv) If $t$ is a number term and $T$ a set term, then $[\sim](t \in T)$ is formula of $L_{R A_{\alpha}}$.

(v) The formulas of $L_{R A_{\alpha}}$ are closed under $\wedge, \vee, \forall x, \exists x, \forall X^{\beta}, \exists X^{\beta}$ for $\beta>0$. 
The level of a set term $T$ and the level of a formula $A$ of $L_{R A_{\alpha}}$ is defined by $\operatorname{lev}(T):=\max \left\{0, \beta: X^{\beta}\right.$ occurs in $\left.T\right\}$ and $\operatorname{lev}(A):=\max \left\{0, \beta: X^{\beta}\right.$ occurs in $\left.A\right\}$.

The rank rk $(A)$ of a formula $A$ of $\mathrm{L}_{\mathrm{RA}_{\alpha}}$ is inductively defined as follows: If $A$ contains no set terms, then $\operatorname{rk}(A):=0$. Otherwise, letting $\mathscr{Q}$ range over $\{\forall, \exists\}$ :

(i) For each set variable $X^{\beta}, \mathrm{rk}\left(t \in X^{\beta}\right):=\operatorname{rk}\left(t \notin X^{\beta}\right):=\max \{1, \omega \cdot \beta\}$,

(ii) $\mathrm{rk}([\sim](s \in\{x: A(x)\})):=\operatorname{rk}(A(0))+1$,

(iii) $\operatorname{rk}(A \vee B)=\operatorname{rk}(A \wedge B):=\max \{\operatorname{rk}(A), \operatorname{rk}(B)\}+1$,

(iv) $\mathrm{rk}(\mathscr{Q} x A(x)):=\mathrm{rk}(A(0))+1$,

(v) $\operatorname{rk}\left(\mathscr{Q} X^{\beta} A\left(X^{\beta}\right)\right):=\max \left\{\omega \cdot \operatorname{lev}\left(\forall X^{\beta} A\left(X^{\beta}\right)\right), \operatorname{rk}\left(A\left(X^{0}\right)\right)+1\right\}$.

Notice that $\mathrm{rk}(A)=\operatorname{rk}(\neg(A))$. Also, if $\operatorname{lev}(A)=\gamma$ and $\operatorname{lev}(T)<\gamma$, then we have $\omega \cdot \gamma \leq \mathrm{rk}(A)<\omega(\gamma+1)$ and $\mathrm{rk}(A(T))<\mathrm{rk}\left(\mathscr{Q} X^{\gamma} A\left(X^{\gamma}\right)\right)$. These properties lead to the partial cut elimination Lemma 7.1.

The semi-formal system $R A_{\alpha}$ is formulate in the language $L_{R A_{\alpha}}$. The formulas of $\mathrm{RA}_{\alpha}$ are the closed formulas of $\mathrm{LRA}_{\alpha}$. Thereby we consider the variable $x$ to occur bound in the set term $\{x: A(x)\}$ and the formula $t \in\{x: A(x)\}$. In order to state the axioms and rules of $\mathrm{RA}_{\alpha}$, we assign to each closed number term $t$ of $\mathrm{L}_{1}$ its value $t^{\mathbb{N}}$ in the standard model. The true literals of $L_{1}$ are the closed literals of $L_{1}$ that evaluate to true in the standard model. The axioms and rules of $\mathrm{RA}_{\alpha}$ are listed below.

Logical axioms. For all finite sets $\Gamma$ of $\mathrm{RA}_{\alpha}$ formulas, all set variables $X^{\beta}$, all true literals $A$ of $L_{1}$ and all closed number terms $s, t$ with $s^{\mathbb{N}}=t^{\mathbb{N}}$ :

$$
\Gamma, A \quad \text { and } \quad \Gamma, t \in X^{\beta}, s \notin X^{\beta} \quad \text { and } \quad \Gamma, t \in \mathrm{D}, s \notin \mathrm{D} \text {. }
$$

Set term rules. For all finite sets $\Gamma$ of $\mathrm{RA}_{\alpha}$ formulas, all formulas $A$ of $\mathrm{RA}_{\alpha}$ and all closed number terms $t$ :

$$
\frac{\Gamma, A(t)}{\Gamma, t \in\{x: A(x)\}}, \quad \frac{\Gamma, \neg A(t)}{\Gamma, t \notin\{x: A(x)\}} .
$$

Quantifier rules. For all finite sets $\Gamma$ of $\mathrm{RA}_{\alpha}$ formulas, all formulas $A$ of $\mathrm{RA}_{\alpha}$, all closed number terms $t$ and all set terms $T$ :

$$
\begin{gathered}
\frac{\Gamma, A(t)}{\Gamma, \exists x A(x)}, \quad \frac{\Gamma, A(s) \text { for all closed number terms } s}{\Gamma, \forall x A(x)} . \\
\frac{\Gamma, A(T) \text { and } \operatorname{lev}(T)<\beta}{\Gamma, \exists X^{\beta} A\left(X^{\beta}\right)}, \quad \frac{\Gamma, A(T) \text { for all set terms with lev }(T)<\beta}{\Gamma, \forall X^{\beta} A\left(X^{\beta}\right)} .
\end{gathered}
$$

D-axioms. For all finite sets $\Gamma$ of $\mathrm{RA}_{\alpha}$ formulas and all closed number terms $t \triangleleft \alpha$ :

$$
\Gamma,\left(\mathrm{Ax}_{\Sigma_{1}^{1}-\mathrm{AC}}\right)^{\mathrm{D}_{t}} \quad \text { and } \Gamma, \mathrm{D}_{\triangleleft t} \dot{\in} \mathrm{D}_{t} .
$$

Rules for $\wedge$ and $\vee$ and cut rules. The usual Tait-style rules for $\wedge$ and $\vee$ as well as the cut rules.

Observe that the D-axioms imply the existence of closed number terms $s$ and $t$, such that $\mathrm{U}=\left(\mathrm{D}_{0}\right)_{t}$ and $\mathrm{V}=\left(\mathrm{D}_{0}\right)_{s}$. Also partial cut elimination is available:

LEMMA 7.1. We have for all finite sets $\Gamma$ of $\mathrm{RA}_{\alpha}$ formulas and all ordinals $\rho>0$ :

(i) $\operatorname{RA}_{\alpha} \frac{\beta}{\rho+1} \Gamma \Longrightarrow \operatorname{RA}_{\alpha} \frac{\omega^{\beta}}{\rho} \Gamma$,

(ii) $\mathrm{RA}_{\alpha} \frac{\beta}{1+\gamma+\omega^{\rho}} \Gamma \Longrightarrow \mathrm{RA}_{\alpha} \frac{\varphi \rho \beta}{1+\gamma} \Gamma$. 
If a finite set $\Gamma$ of $L_{2}(D)$ formulas is provable in $\left(M_{\alpha}+A C A\right)_{0}$, then standard cut elimination techniques yield that it is already provable in $\left(M_{\alpha}+A C A\right)_{0}$ where the cut rule is restricted to cut formulas $A$ that are either elementary or $[\neg] A$ is the main formula of an axiom for arithmetical comprehension. Such restricted derivations are denoted by $\left.\left(M_{\alpha}+A C A\right)_{0}\right|_{*} \Gamma$.

LEMMA 7.2. Let $\Gamma(\vec{X}, \vec{x})$ be a finite set of elementary formulas of $L_{2}(D)$ such that $\left(\mathrm{M}_{\alpha}+\mathrm{ACA}\right)_{0} \vdash_{*} \Gamma(\vec{X}, \vec{x})$. Then there exists for all set terms $\vec{S}$ of level 0 an ordinal $\beta<\omega \cdot \max \{\alpha, \omega\}+\omega$ such that for all closed number terms $\vec{s}$,

$$
\mathrm{RA}_{\alpha} \frac{\beta}{<\omega} \Gamma(\vec{S}, \vec{s}) \text {. }
$$

ProOF. We proof the claim by induction on the length of the proof in $\left(\mathrm{M}_{\alpha}+\mathrm{ACA}\right)_{0}$. For a set term $S$ of level $0, \operatorname{RA}_{\alpha} \frac{<\omega \cdot \max \{\alpha, \omega\}+\omega}{<\omega} \operatorname{TI}(S, \max \{\alpha, \omega\})$, and similarly for the other elementary main formulas of an axiom of $M_{\alpha}$. The only case that follows not directly from the I.H. is if the last inference was a cut with a cut formula of the form $\exists X \forall x[x \in X \leftrightarrow A(\vec{Y}, x)]$ for some arithmetical formula $A$, or $t \triangleleft \alpha \rightarrow \exists X\left[X=\mathrm{D}_{a}\right]$. We just consider the first case: $\forall$-inversion and the I.H. yield that for arbitrary set terms $\vec{S}$ of level 0 and $T:=\{x: A(\vec{S}, x)\}$, there is a $\beta<\omega \cdot \max \{\alpha, \omega\}+\omega$ such that for all closed number terms $\vec{s}$,

$$
\mathrm{RA}_{\alpha} \vdash_{<\omega}^{\beta} \Gamma(\vec{S}, \vec{s}), \neg \forall x[x \in T \leftrightarrow A(\vec{S}, x)] .
$$

Because of RA $\left.\right|_{\alpha}|<\omega|<x[x \in T \leftrightarrow A(\vec{S}, x)]$, a cut yields the claim.

Now we move to the theory $\left(M_{\alpha}+\Sigma_{1}^{1}-D C\right)_{0}$. Cantini has shown in [5] that there is an asymmetric interpretation of $\Sigma_{1}^{1}-D C_{0}$ into $\Pi_{0}^{1}-C A_{<\omega^{\omega}}$. The same proof allows to perform an asymmetric interpretation of the theory $\left(M_{\alpha}+\Sigma_{1}^{1}-D C\right)_{0}$ into $\left(M_{\alpha}+H_{i e r}^{J} \omega^{\omega}\right)_{0}$, which extends $M_{\alpha}$ by an axiom asserting the existence of the jump-hierarchy above any set $X$ along an initial segment of $\triangleleft$ of ordertype less then $\omega^{\omega}:$
$\left(\right.$ Hier $\left._{<\omega^{\omega}}^{J}\right)$
$\Gamma, \exists F \operatorname{Hier}^{\jmath}(F, X, \beta)$,

for each ordinal $\beta<\omega^{\omega}$ and finite sets $\Gamma$ of $\mathrm{L}_{2}(\mathrm{D})$ formulas, where $\operatorname{Hier}^{\mathrm{J}}(F, X, a)$ denotes the formula

$$
(\forall b \triangleleft a)\left[(F)_{b}=\left\{\langle x,\langle c, e\rangle\rangle: \pi_{1}^{0}\left(\left((F)_{\triangleleft b}\right)_{c}, X, e, x\right)\right\}\right],
$$

expressing that $F$ constitutes a jump-hierarchy above $X$ along $a \in \operatorname{Field}(\triangleleft)$. For more details on this particular definition of the jump-hierarchy, we refer to [14].

LEMMA 7.3. $\left(\mathrm{M}_{\alpha}+\Sigma_{1}^{1}-\mathrm{DC}\right)_{0}$ and $\left(\mathrm{M}_{\alpha}+\mathrm{Hier}_{<\omega^{\omega}}^{\mathrm{J}}\right)_{0}$ prove the same $\Pi_{2}^{1}$ formulas of $\mathrm{L}_{2}$.

If $\left(M_{\alpha}+H_{i e r}^{J}{ }_{<\omega^{\omega}}\right)_{0} \vdash \Gamma$, then there is already a derivation $\left(M_{\alpha}+H_{i e r}^{J}{ }_{<\omega^{\omega}}\right)_{0} \downarrow_{*} \Gamma$ which only uses the cut rule for elementary formulas and main formulas of instances of arithmetical comprehension and ( Hier $_{<\omega^{\omega}}^{J}$ ). For each $\beta, \gamma<\omega^{\omega}$ and each set terms $S$ with level $\operatorname{lev}(S)=\gamma$, there is a set term $T$ of level $<\omega^{\omega}$ such that

$$
\mathrm{RA}_{\alpha} \frac{<\omega^{\omega}}{<\omega^{\omega}} \operatorname{Hier}(T, S, \beta) \text {. }
$$

Similar as before, we obtain the following lemma. 
LEMMA 7.4. Let $\Gamma(\vec{X}, \vec{x})$ be a finite set of elementary formulas of $\mathrm{L}_{2}(\mathrm{D})$ such that $\mathrm{M}_{\alpha}+\left(\mathrm{Hier}_{<\omega^{\omega}}^{\mathrm{J}}\right) \vdash_{*} \Gamma(\vec{X}, \vec{x})$. Then there exists for all set terms $\vec{S}$ of level less than $\omega^{\omega}$ an ordinal $n<\omega$ such that for all closed number terms $\vec{s}$

$$
\mathrm{RA}_{\alpha} \stackrel{<\omega \cdot \alpha+\omega^{n}}{<\omega^{n}} \vdash \Gamma(\vec{S}, \vec{s}) \text {. }
$$

Collecting the previous results and applying partial cut elimination for $\mathrm{RA}_{\alpha}$ yields the following:

Lemma 7.5. Suppose that $A$ is a sentence of $L_{1}$. Then we have:

(i) $\left(\mathrm{M}_{\alpha}+\mathrm{ACA}\right)_{0} \vdash A \Longrightarrow \mathrm{RA}_{\alpha} \vdash \frac{<\varepsilon_{0}(\alpha)}{1} A$,

(ii) $\left(\mathrm{M}_{\alpha}+\Sigma_{1}^{1}-\mathrm{DC}\right)_{0} \vdash A \Longrightarrow \mathrm{RA}_{\alpha} \vdash \frac{<(\alpha \mid 1)}{1} A$.

Next, we want to reduce $\mathrm{RA}_{\alpha}$ to the semi-formal system $\mathrm{E}_{\alpha}^{0}$. Basically, $\mathrm{E}_{\alpha}^{0}$ corresponds to the first order part of $\mathrm{RA}_{\alpha}$. Due to Rüede's results in [15], a prove of an $\mathrm{L}_{1}$ formula $A$ in $\mathrm{E}_{\alpha}^{0}$ yields a cut-free derivation of $A$ in $\mathrm{E}_{\alpha}^{0}$, which corresponds to a derivation of $A$ in PA* $^{*}$, a Tait-style reformulation of Peano Arithmetic PA with $\omega$-rule.

For the reader's convenience, we restate Rüede's system $E_{\alpha}^{0}$. The language of $E_{\alpha}^{0}$ is the extension of $L_{1}$ by unary relation symbols $D_{\beta}^{0}$ and $D_{<\gamma}^{0}$ for each $\beta<\alpha$ and $\gamma \leq \alpha$. The formulas of $E_{\alpha}^{0}$ are the formulas of the language of $E_{\alpha}^{0}$ that do not contain free number variables.

The ontological axioms and rules of $\mathrm{E}_{\alpha}^{0}$ state that for $\beta<\alpha, \mathrm{D}_{\beta}^{0}$ contains only pairs, i.e., if the closed number term $t$ is an element of $D_{\beta}^{0}$, then its value is $\langle m, n\rangle$ for some natural numbers $m, n$. This expresses that $m$ is an element of the set with code $n$ in $\mathrm{D}_{\beta}^{0}$. The closure axioms and rules express that for all $\beta<\alpha, \mathrm{D}_{\beta}^{0}$ is a model of $\Sigma_{1}^{1}-A C$ and that $D_{<\beta}^{0} \in \mathrm{D}_{\beta}^{0}$.

Logical axioms of $\mathrm{E}_{\alpha}^{0}$. For all finite sets $\Gamma$ of $\mathrm{E}_{\alpha}^{0}$ formulas, all true literals $A$ of $L_{1}$ and all closed number terms $s, t$ with identical values and all ordinals $\beta<\alpha, \gamma \leq \alpha$ :

$$
\Gamma, A \quad \text { and } \quad \Gamma, t \in \mathrm{D}_{\beta}^{0}, s \notin \mathrm{D}_{\beta}^{0} \quad \text { and } \quad \Gamma, t \in \mathrm{D}_{<\gamma}^{0}, s \notin \mathrm{D}_{<\gamma}^{0} .
$$

Ontological axioms and rules of $\mathrm{E}_{\alpha}^{0}$. For all finite sets $\Gamma$ of formulas of $\mathrm{E}_{\alpha}^{0}$, all $\beta \leq \alpha$, $\gamma<\beta$, all closed number terms $r, s, t$ such that $r$ is not a pair, $s$ is a pair but $(s)_{0}$ is not a pair and $\beta \unlhd(s)_{1}$, and $t$ is a pair and $\gamma=(t)_{1}$,

$$
\Gamma, r \notin \mathrm{D}_{<\beta}^{0}, \quad \Gamma, s \notin \mathrm{D}_{<\beta}^{0}, \quad \frac{\Gamma,(t)_{0} \in \mathrm{D}_{\gamma}^{0}}{\Gamma, t \in \mathrm{D}_{<\beta}^{0}}, \quad \frac{\Gamma,(t)_{0} \notin \mathrm{D}_{\gamma}^{0}}{\Gamma, t \notin \mathrm{D}_{<\beta}^{0}} .
$$

D-axioms of $\mathrm{E}_{\alpha}^{0}$. For all finite sets $\Gamma$ of $\mathrm{E}_{\alpha}^{0}$ formulas, all closed number terms $e, r, s, t$ and all ordinals $\beta<\alpha$ :

$$
\begin{gathered}
\Gamma, \exists k\left[\left(\mathrm{D}_{\beta}^{0}\right)_{k}=\left(\mathrm{D}_{\beta}^{0}\right)_{t} \oplus\left(\mathrm{D}_{\beta}^{0}\right)_{s}\right], \\
\Gamma, \exists k\left[\forall x\left(x \in\left(\mathrm{D}_{\beta}^{0}\right)_{k} \leftrightarrow \pi_{1}^{0}\left(\left(\mathrm{D}_{\beta}^{0}\right)_{t}, \mathrm{D}_{<\beta}^{0}, e, x, r\right)\right] .\right.
\end{gathered}
$$

D-rules of $\mathrm{E}_{\alpha}^{0}$. For all finite sets $\Gamma$ of $\mathrm{E}_{\alpha}^{0}$ formulas, all closed number terms $e, r, s, t$ and all ordinals $\beta<\alpha$ :

$$
\frac{\Gamma, \forall x \exists k \pi_{2}^{0}\left(\left(\mathrm{D}_{\beta}^{0}\right)_{k},\left(\mathrm{D}_{\beta}^{0}\right)_{t}, \mathrm{D}_{<\beta}^{0}, e, x, r\right)}{\Gamma, \exists k \forall x \pi_{2}^{0}\left(\left(\left(\mathrm{D}_{\beta}^{0}\right)_{k}\right)_{x},\left(\mathrm{D}_{\beta}^{0}\right)_{t}, \mathrm{D}_{<\beta}^{0}, e, x, r\right)} .
$$


Propositional rule, rules for the first order quantifiers and cut rules. These are the rules for $R A_{\alpha}$ adapted to the language of $E_{\alpha}^{0}$.

For a precise definition of the rank of formulas of $E_{\alpha}^{0}$ we refer to definition 11 and the subsequent paragraph in [15]. We just try to capture the general idea: For example, if $\beta<\alpha$ and $A(X)$ is a formula of $L_{2}$ with exactly the displayed set variable free, then $\left(\forall X \dot{\in} \mathrm{D}_{\beta}^{0}\right) A(X),\left(\exists X \dot{\in} \mathrm{D}_{<\beta}^{0}\right) A(X),\left(\forall X \dot{\in} \mathrm{D}_{\beta}^{0}\right) A(X)$ and $\left(\exists X \dot{\in} \mathrm{D}_{<\beta}^{0}\right) A(X)$ are formulas of the language of $\mathrm{E}_{\alpha}^{0}$ of rank zero. Further, formulas of the language of $E_{\alpha}^{0}$ of rank zero are closed under number quantification and propositional connectives. If $\beta+1=\alpha$ and $t$ is a closed term that is not a pair, then $t \in \mathrm{D}_{\beta}^{0}$ has rank 1. Also $t \in \mathrm{D}_{<\alpha}^{0}$ has rank 1. Moreover, the rank of an $\mathrm{E}_{\alpha}^{0}$ formula is always finite and the rank of all main formulas of axioms of $E_{\alpha}^{0}$ is zero.

LEMMA 7.6. For all natural numbers $n>0$ we have:

$$
\left.\mathrm{E}_{\alpha}^{0} \frac{\beta}{n+1} \Gamma \Longrightarrow \mathrm{E}_{\alpha}^{0}\right|^{2^{\beta}} \frac{}{n} \Gamma
$$

A closed $L_{1}(D)$ formula $A$ is translated to an formula $A^{D_{<\alpha}^{0}}$ of $E_{\alpha}^{0}$ by simply replacing $\mathrm{D}$ by $\mathrm{D}_{<\alpha}^{0}$ in $A$. Now the following lemma, which corresponds to $\mathrm{c}$ ) of Theorem 20 in [15], yields an embedding of $\mathrm{RA}_{\alpha}$ into $\mathrm{E}_{\alpha}^{0}$.

LEMMA 7.7. Let $\alpha<\Phi_{0}$ and $\Gamma$ a finite set of closed formulas of $L_{1}(D)$. Then we have:

$$
\left.\left.\mathrm{RA}_{\alpha}\right|_{\mathrm{T}} ^{\delta} \Gamma \Longrightarrow \mathrm{E}_{\alpha}^{0}\right|^{<\omega \cdot \delta} \Gamma^{\mathrm{D}_{<a}^{0}}
$$

Proor. As in [15] this follows by an induction on $\delta$. Since in our case, the Daxioms and D-rules of $E_{\alpha}^{0}$ are syntactically different from the $D$-axioms of $R A_{\alpha}$, we have to use that for a closed number term $t \triangleleft \alpha$ with $t=\beta, \mathrm{E}_{\alpha}^{0} \frac{<\omega}{0}\left(\mathrm{D}_{<\alpha}^{0}\right)_{t}=\mathrm{D}_{\beta}^{0}$ and $\left.\mathrm{E}_{\alpha}^{0}\right|_{<\omega} ^{<\omega}\left(\mathrm{Ax}_{\Sigma_{1}^{1}-\mathrm{AC}}\right)^{\left(D_{<\alpha}^{0}\right)_{t}}$.

For $M_{\lambda} \uparrow$, the detour over $R A_{\lambda}$ is not necessary. We first embed $M_{\lambda} \uparrow$ into $E_{\lambda}^{+}$, the extension of $E_{\lambda}^{0}$ by axioms

$$
\Gamma,\left(\forall X \dot{\in} \mathrm{D}_{\beta}\right) \mathrm{TI}_{\triangleleft}(X, \lambda),
$$

for each $\beta<\lambda$ and all finite sets $\Gamma$ of $L_{1}(D)$ formulas.

LEMMA 7.8. Let $\lambda<\Phi_{0}$ and $\Gamma(\vec{u})$ a finite set of formulas of $L_{1}(D)$. Then there exists for each $n \in \mathbb{N}$ an $n^{\prime} \in \mathbb{N}$, such that we have for all closed number terms $\vec{t}$,

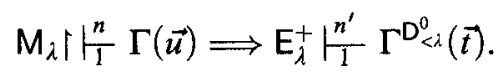

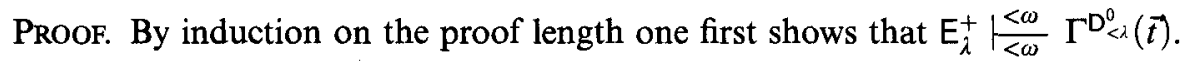
Then cut elimination in $E_{\lambda}^{+}$yields the claim.

Theorem 26 in [15] tells us at what cost we can reduce $E_{\beta+\omega^{1+\rho}}^{0}$ to $E_{\beta}^{0}$. Given this result, the reduction of $\mathrm{E}_{\beta+\omega^{1+\rho}}^{+}$to $\mathrm{E}_{\beta}^{0}$ does not cause additional difficulties.

LEMmA 7.9. Assume that $\lambda=\beta+\omega^{1+\rho}<\Phi_{0}, \lambda\left\lceil 0=\lambda_{0}+\omega\right.$ and $k$ is the least natural number such that $\varphi 1 \rho\left(\lambda_{0}+k\right)>\lambda$. Further, suppose that $\left.\mathrm{E}_{\lambda}^{+}\right|_{1} ^{n} \Gamma$ for a finite set $\Gamma$ of formulas of $\mathrm{E}_{\beta+\omega^{1+\rho}}^{0}$ of rank 0 . Then the following holds: If each formula in $\Gamma$ is a formula of $\mathrm{E}_{\beta+\xi^{\prime}}^{0}$ for some $\xi^{\prime}<\xi<\omega^{1+\rho}$, then

$$
\mathrm{E}_{\beta+\xi}^{0} \frac{\varphi 1 \rho\left(\lambda_{0}+k+n\right)}{1} \Gamma \text {. }
$$


ProOF. The lemma is proved as Theorem 26 in [15] by main induction on $\rho$ and side induction on $n$. For $n=0$, observe that $\gamma<\xi<\omega^{1+\rho}$ yields

$$
\mathrm{E}_{\beta+\xi}^{0} \frac{<\varphi 1 \rho\left(\lambda_{0}+k\right)}{1}\left(\forall X \dot{\in} \mathrm{D}_{\gamma}^{0}\right) \mathrm{TI}_{\triangleleft}(X, \lambda) .
$$

Now we conclude that the theories $\left(M_{\alpha}+A C A\right)_{0},\left(M_{\alpha}+\Sigma_{1}^{1}-D C\right)_{0}$ and $M_{\lambda} \uparrow$ have the desired upper bounds.

THEOREM 7.10 (Upper Bounds). For $\alpha<\Phi_{0}$ and limit ordinals $\lambda<\Phi_{0}$, we have:

$$
\left|\left(\mathrm{M}_{\alpha}+\mathrm{ACA}\right)_{0}\right| \leq \sigma(\alpha), \quad\left|\left(\mathrm{M}_{\alpha}+\Sigma_{1}^{1}-\mathrm{DC}\right)_{0}\right| \leq \sigma\left\lceil(\alpha+1) \quad \text { and } \quad\left|\mathrm{M}_{\lambda}\right||\leq \sigma|(\lambda)\right. \text {. }
$$

Proof. Suppose that $\alpha=\omega^{1+\alpha_{n}}+\cdots+\omega^{1+\alpha_{1}}+m$, where $\alpha_{n} \geq \cdots \geq \alpha_{1}$ and $m<\omega$, and let $\delta$ and $\lambda$ such that $\alpha=\lambda+m$ and $\lambda=\delta+\omega^{1+\alpha_{1}}$. Further, assume that $A$ is a sentence of $\mathrm{L}_{1}$. If $\left(\mathrm{M}_{\alpha}+\mathrm{ACA}\right)_{0} \vdash A$, then the Lemmas 7.5, 7.6 and 7.7 yield $\mathrm{E}_{\alpha}^{0} \vdash \frac{<(\alpha \mid 0)}{1} A$, so that applying $m$-times Corollary 21 in [15] gives $\mathrm{E}_{\lambda}^{0} \vdash \frac{<(\alpha \mid m)}{1} A$. Now $n$-fold application of Theorem 26 in [15] confirms $\mathrm{E}_{0}^{0} \frac{<\sigma(\alpha)}{1} A$. Similarly, if $\left(\mathrm{M}_{\alpha}+\Sigma_{1}^{1}-\mathrm{DC}\right)_{0} \vdash A$, then $\mathrm{E}_{\alpha}^{0} \frac{<(\alpha\lceil 1)}{1} A$, thus we obtain $\mathrm{E}_{\lambda}^{0} \frac{<(\alpha \mid m+1)}{1} A$ and $\mathrm{E}_{0}^{0} \frac{<\sigma\lceil(\alpha+1)}{1} A$. Finally, if $\mathrm{M}_{\lambda} \uparrow \vdash A$, then $\mathrm{E}_{\lambda}^{+} \frac{{ }^{<\omega}}{1} A$ due to Lemma 7.8 , therefore $\mathrm{E}_{\delta}^{0} \frac{<\varphi 1 \alpha_{n}(\lambda\lceil 0)}{1} A$ and $\mathrm{E}_{0}^{0} \frac{<\sigma\lceil(\lambda)}{1} A$. Cut-elimination in $\mathrm{PA}^{*}$ yields the claim.

7.2. Embedding the $I D^{*}$-theories into the $M^{\dagger}$-theories. Let $M$ denote one of the $M$-theories and let $\xi$ be the upper bound according to Theorem 7.10. Note that for $\beta<\xi$ the ordinal $\omega \cdot \beta$ is still less than $\xi$. By choice of $\xi$, we have that $\mathrm{TI}_{\triangleleft}(\mathrm{V}, \xi)$ is not provable in the theory $M$. Therefore, the theory $M^{\dagger}$, the extension of $M$ by the axiom $\neg \mathrm{TI}_{\triangleleft}(\mathrm{V}, \xi)$, is consistent. Moreover, $\xi$ is still an upper bound of $\mathrm{M}^{\dagger}$ : Assume that $\mathrm{M}^{\dagger}$ proves $\mathrm{TI}_{\prec}(\mathrm{U}, \alpha)$ for a primitive recursive well-ordering $\prec$. Thus $\mathrm{M} \vdash \mathrm{TI}_{\triangleleft}(\vee, \xi) \vee \mathrm{TI}_{\prec}(\mathrm{U}, \alpha)$. The proof of Theorem 7.10 yields that

$$
\mathrm{PA}^{*} \frac{<\xi}{0} \neg \mathrm{Prog}_{\triangleleft}(\mathrm{V}), \xi \in \mathrm{V}, \mathrm{TI} \mathrm{l}_{\prec}(\mathrm{U}, \alpha)
$$

With Lemma 4 in Jäger and Probst [10] we conclude that also $\left.\mathrm{PA}^{*}\right|_{0} ^{<\xi} \mathrm{TI}_{\prec}(\mathrm{U}, \alpha)$. Hence, by Schütte's boundedness theorem (cf. [16] or [12] ) we obtain $\alpha<\xi$. Embedding the $I D^{*}$-theory with lower bound $\xi$ into $\mathrm{M}^{\dagger}$, then yields $\xi \leq\left|\mathrm{ID}^{*}\right| \leq\left|\mathrm{M}^{\dagger}\right| \leq \xi$.

To embed the $\mathrm{ID}^{*}$-theories into the $\mathrm{M}^{\dagger}$-theories, we show that these theories prove the existence of $\alpha$-hierarchies of models of $\Sigma_{1}^{l}-D C$. Thereby, we make use of so-called pseudo-hierarchy arguments. For second order arithmetic, this method is described in Simpson [18] in extenso and a typical application is given in Avigad [2]. In subsystems of second order arithmetic comprising (ACA), the existence of a pseudo-hierarchy follows from the fact that being a well-ordering is not expressible by a $\Sigma_{1}^{1}$ formula of $L_{2}$. However, this method does not provide uniform pseudohierarchies.

We apply a more general method to obtain pseudo-hierarchies: Due to the axiom $\neg \mathrm{TI}_{\triangleleft}(\mathrm{V},|\mathrm{M}|)$ of the theory $\mathrm{M}_{\alpha}^{\dagger}$ one can prove that $\left\{a \in \operatorname{Field}(\triangleleft): \forall Z \mathrm{TI}_{\triangleleft}(Z, a)\right\}$ is not a set. The existence of [uniform] pseudo-hierarchies is then derived from this observation. Using this method, the application of pseudo-hierarchy arguments is no longer limited to second order analysis and can be applied in the context of explicit mathematics and admissible set theory as well; cf. [13, 14]. In the sequel, 
$W_{\triangleleft}(a)$ is to abbreviate $\forall Z \mathrm{TI}_{\triangleleft}(Z, a)$ and $\operatorname{Hier}^{\mathrm{J}}(U, V, u)$ is the formula defined above Lemma 7.3.

LEMMA 7.11. The following is provable in $\left(\mathrm{M}_{\alpha}+\mathrm{ACA}\right)_{0}^{\dagger}$ :

(i) $\left\{a: W_{\triangleleft}(a)\right\}$ is not a set.

(ii) If $b \triangleleft \alpha$, then $\left\{a: W_{\triangleleft}^{\mathrm{D}_{h}}(a)\right\} \notin \mathrm{D}_{b}$.

(iii) If $b+1 \triangleleft \alpha$ and $X \dot{\in} \mathrm{D}_{b}$, then $\left\{a: \mathrm{Wo}_{\triangleleft}^{\mathrm{D}_{b+1}}(a)\right\} \subsetneq\left\{a:\left(\exists F \dot{\in} \mathrm{D}_{b}\right) \operatorname{Hier}^{\mathrm{J}}(F, X, a)\right\}$.

Proof. Suppose for a moment that $S:=\left\{a: W_{0_{\triangleleft}}(a)\right\}$ is a set. Then the set $S_{0}:=\{a:(\forall b \in S)(\varphi 0 a b \in S)\}$ is easily shown to be progressive w.r.t. $\triangleleft$, which in turn yields the progressivity of the set $S_{1}:=\{a:(\forall b \in S)(\varphi 1 a b \in S)\}:$ If $a=0$, this is due to the progressivity of $S_{0}$, otherwise assume that $\left(\forall a^{\prime} \triangleleft a\right)\left(a^{\prime} \in S_{1}\right)$ and show that $\operatorname{Prog}_{\triangleleft}(\{b \in S: \varphi 1 a b \in S\})$, which yields $a \in S_{1}$. Hence $a, b \in S$ implies $\varphi 1 a b \in S$. In particular, $\alpha \in S$ yields $\sigma(\alpha) \in S$. A contradiction!

If we relativize the above argument to $\mathrm{D}_{b}$, we obtain that $\sigma(\alpha) \in\left\{a: \mathrm{Wo}_{\triangleleft}^{\mathrm{D}_{b}}(a)\right\}$. Since $\mathrm{V} \in \mathrm{D}_{b}$, this contradicts $\neg \mathrm{TI}_{\triangleleft}(\mathrm{V}, \sigma(\alpha))$. Thus (ii) holds.

Because $\mathrm{D}_{b}$ is a model of $\Sigma_{1}^{1}-\mathrm{AC}, \mathrm{Wo}_{\triangleleft}^{\mathrm{D}_{h+1}}(a)$ implies the existence of an $F \dot{\in} \mathrm{D}_{b}$ such that $\operatorname{Hier}^{\mathrm{J}}(F, X, a)$. Because $\left\{a: \mathrm{Wo}_{\triangleleft}^{\mathrm{D}_{b+1}}(a)\right\}$ is not a set in $\mathrm{D}_{b+1}$, the inclusion is proper.

LEMMA 7.12. The following is provable in $\left(\mathrm{M}_{\alpha+1}+\mathrm{ACA}\right)_{0}^{\dagger}$ : For each set $X \dot{\in} \mathrm{D}_{\alpha}$ there exists a model $M$ of $\Sigma_{1}^{1}-\mathrm{DC}$ with $X \dot{\in} M$.

Proof. Fix $X \dot{\in} \mathrm{D}_{\alpha}$. Since $\mathrm{D}_{\alpha}$ is a model of $\Sigma_{1}^{1}-\mathrm{AC}$, one easily proves that

$$
\forall a\left[\mathrm{Wo}_{\triangleleft}(a) \rightarrow\left(\exists F \dot{\in} \mathrm{D}_{\alpha}\right) \operatorname{Hier}^{\lrcorner}(F, X, a) \wedge \mathrm{Wo}_{\triangleleft}^{\mathrm{D}_{\alpha}}(a)\right] .
$$

Because $\left\{a: W_{\triangleleft}(a)\right\}$ is not a set, there exists a $b \in \operatorname{Field}(\triangleleft)$ and an $F \dot{\in} \mathrm{D}_{\alpha}$ such that

$$
\neg \mathrm{Wo}_{\triangleleft}(b) \wedge \operatorname{Hier}^{\mathrm{J}}(F, X, b) \wedge \mathrm{Wo}_{\triangleleft}^{\mathrm{D}_{\alpha}}(b),
$$

Thus, there exists a non-empty, upward closed $K \subseteq$ Field $(\triangleleft)$ without a $\triangleleft$-least element and with $b \in K$. Surely, Wo $\triangleleft(a)$ implies $(\forall x \in K)(a \triangleleft x)$, subsequently abbreviated by $a \triangleleft K$. Next we consider the sets

$$
\begin{aligned}
M:= & M_{\triangleleft K}^{F}:=\left\{\langle x,\langle c, e\rangle\rangle: c \triangleleft K \wedge\langle x,\langle c, e\rangle\rangle \in(F)_{c+1}\right\}, \\
M_{\triangleleft d}^{F}: & =\left\{\langle x,\langle c, e\rangle\rangle: c \triangleleft d \wedge\langle x,\langle c, e\rangle\rangle \in(F)_{c+1}\right\}, \text { for each } d \in \text { Field }(\triangleleft),
\end{aligned}
$$

and prove that $M$ is a model of $\Sigma_{1}^{1}$-DC. We just show that $M$ satisfies $\left(\Sigma_{1}^{1}-\mathrm{DC}\right)$, that $M$ is a model of ACA follows from standard results concerning the jump-hierarchy. So, let $A(U, V)$ be an arithmetical formula of $\mathrm{L}_{2}$ and assume that

$$
(\forall X \dot{\in} M)(\exists Y \dot{\in} M) A(X, Y) .
$$

If $X \dot{\in} M$, then there exists an index $a$ such that $X=(M)_{a}$. The definition of $M$ implies that $a$ is of the form $\langle c, e\rangle$, where $e$ is a natural number and $c$ an element of the field of $\triangleleft$. Now, we set

$$
\mathrm{I}:=\{\langle c, e\rangle: e \in \mathrm{N} \wedge c \in \text { Field }(\triangleleft)\},
$$

and order $\mid$ by $<_{1}$, letting $\langle c, e\rangle<_{1}\left\langle d, e^{\prime}\right\rangle$ if $c \triangleleft d$, or $c=d$ and $e<_{N} e^{\prime}$. Note, that $\langle c, e\rangle \in \mathrm{I}$ and $\neg(c \triangleleft K)$ implies $(M)_{\langle c, e\rangle}=\emptyset$. Therefore, (1) becomes equivalent 
to the formula $(\forall y \in I)(\exists z \in 1) A\left((M)_{y},(M)_{z}\right)$. Moreover, for each $y \in 1$, the set $\left\{z \in \mathrm{I}: A\left((M)_{y},(M)_{z}\right)\right\}$ has a $<$-least element. To see this, observe that

$$
S_{1}:=\left\{z \in \mathrm{I}: A\left((M)_{y},(M)_{z}\right)\right\} \subseteq\left\{z \in \mathrm{I}: A\left(\left(M_{\triangleleft b}^{F}\right)_{y},\left(M_{\triangleleft b}^{F}\right)_{z}\right)\right\}=: S_{2} .
$$

Since $S_{2} \dot{\in} \mathrm{D}_{\alpha}$ and $\mathrm{Wo}_{\triangleleft}^{\mathrm{D}_{\alpha}}(b)$, it has a $\triangleleft$-least element. This is also the minimum of the set $S_{1}$, because $z \in S_{1}, y \in S_{2}$ and $y<_{1} z$ yields already $y \in S_{1}$. Therefore, we conclude that $(\forall y \in I)(\exists ! z \in I) A^{\prime}(M, y, z)$, where $A^{\prime}$ is an arithmetical formula of $\mathrm{L}_{2}$ expressing that $z$ is the least index w.r.t. our index ordering $<_{1}$, such that $A\left((M)_{y},(M)_{z}\right)$ holds.

Next, we fix an index $w \in \mathrm{I}$ with $(w)_{0} \triangleleft K$ and show that there exists a choice sequence $Z \dot{\epsilon} M$, such that $(Z)_{0}=(M)_{w}$ and $\forall n A\left((Z)_{n},(Z)_{n+1}\right)$. First, we look for initial segments of such a choice sequence. In the present setting, this is a finite sequence $s$, (respectively a natural number of the form $\left\langle x_{1}, \ldots, x_{n}\right\rangle$ ) of indices such that

$$
\mathrm{ChSeq}_{A^{\prime}}(M, s, w, n):=\operatorname{lh}(s)=n+1 \wedge(s)_{0}=w \wedge(\forall m<n) A^{\prime}\left(M,(s)_{m},(s)_{m+1}\right) .
$$

Assumption (1) allows us to prove by set induction that $\forall n \exists ! s \mathrm{ChSeq}_{A^{\prime}}(M, s, w, n)$. Further, $c \triangleleft K$ implies $(M)_{\langle c, e\rangle}=\left(M_{\triangleleft a}^{F}\right)_{\langle c, e\rangle}$ for each $a \in K$, thus the set

$$
\left\{a \triangleleft b: \forall n \exists s \operatorname{ChSeq}_{A^{\prime}}\left(M_{\triangleleft a}^{F}, s, w, n\right)\right\}
$$

is not empty. Moreover, it is in $\mathrm{D}_{\alpha}$, so it has a least element $a_{0}$. Since $a_{0} \triangleleft K$,

$$
Z:=\left\{\langle x, n\rangle: \exists s\left[\mathrm{ChSeq}_{A^{\prime}}\left(M_{\triangleleft a_{0}}^{F}, s, w, n\right) \wedge x \in\left(M_{\triangleleft a_{0}}^{F}\right)_{(s)_{n}}\right]\right\}
$$

is a set in $M$ and serves as a witness for our sought for choice sequence.

The model constructed in the previous proof is not uniform in the sense that we only know about the existence of a set $K$ without a $\triangleleft$-least element, but cannot explicitly define it. However, if $X \dot{\in} \mathrm{D}_{b}$ and $b+1 \triangleleft \alpha$, then we can construct in $\left(\mathrm{M}_{\alpha}+\mathrm{ACA}\right)_{0}^{\dagger}$ a uniform model $M$ of $\Sigma_{1}^{1}-\mathrm{DC}$ above $X$. More precisely: If $X \dot{\mathrm{D}} \mathrm{D}_{b}$, the we call the set

$$
M_{\Sigma_{1}^{1}-\mathrm{DC}}(X, b):=\left\{x: \mathrm{Wo}_{\triangleleft}^{\mathrm{D}_{b+1}}(c) \wedge\left(\exists F \dot{\in} \mathrm{D}_{b}\right)\left[\operatorname{Hier}^{\mathrm{J}}(F, X, c+1) \wedge x \in M_{\triangleleft c}^{F}\right]\right\},
$$

the uniform model of $\Sigma_{1}^{1}-\mathrm{DC}$ above $X \dot{\in} \mathrm{D}_{b} . M_{\triangleleft c}^{F}$ is as defined in the above proof. Because $\mathrm{Wo}_{\triangleleft}^{\mathrm{D}_{b+1}}(c)$ and $X \dot{\in} \mathrm{D}_{b}$ imply that there is exactly one $F \dot{\epsilon} \mathrm{D}_{b}$ satisfying $\operatorname{Hier}^{\mathrm{J}}(F, X, c+1)$, the set $M_{\Sigma_{1}^{1}-\mathrm{DC}}(X, b)$ is clearly unique.

LEMMA 7.13. The following is provable in $\left(\mathrm{M}_{\alpha}+\mathrm{ACA}\right)_{0}^{\dagger}:$ If $b+1 \triangleleft \alpha$ and $X \dot{\mathrm{D}} \mathrm{D}_{b}$, then $M_{\Sigma_{1}^{1}-\mathrm{DC}}(X, b)$ is a model of $\Sigma_{1}^{1}-\mathrm{DC}$ with $X \dot{\in} M_{\Sigma_{1}^{1}-\mathrm{DC}}(X, b)$. If $b+2 \triangleleft \alpha$ then $M_{\Sigma_{1}^{1}-\mathrm{DC}}(X, b) \dot{\in} \mathrm{D}_{b+2}$.

Proof. Assume that $X \dot{\in} \mathrm{D}_{b}$. Using (iii) of Lemma 7.11, we can find an $F \dot{\in} \mathrm{D}_{b}$ and a notation $d \in$ Field $(\triangleleft)$ such that $\neg W_{\triangleleft}^{D_{b+1}}(d)$ and $\operatorname{Hier}^{J}(F, X, d)$. Further,

$$
K:=\left\{a \in \text { Field }(\triangleleft): \neg W_{\triangleleft}^{D_{b+1}}(a)\right\},
$$

is a non-empty subset of Field $(\triangleleft)$ without a $\triangleleft$-least element that contains $d$. Now $M_{\Sigma_{1}^{1}-\mathrm{DC}}(X, b)$ becomes the set $M_{\triangleleft K}^{F}$ according to the definition in the previous proof, and we may continue as there. 
Next we want to speak about [uniform] $a$-hierarchies of models of $\Sigma_{1}^{1}$-DC. For this purpose, we set:

$$
\begin{aligned}
& \operatorname{Hier}_{\Sigma_{1}^{\prime}-\mathrm{DC}}(F, a) \Longleftrightarrow(\forall b \triangleleft a)\left[(F)_{b}=M_{\Sigma_{1}^{\prime}-\mathrm{DC}}\left((F)_{\triangleleft b}, 2 b\right)\right], \\
& \operatorname{Hier}_{\Sigma_{1}^{1}-\mathrm{DC}}(F, a): \Longleftrightarrow(\forall b \triangleleft a)\left[(F)_{\triangleleft b} \dot{\in}(F)_{b} \wedge\left(\operatorname{Ax}_{\Sigma_{1}^{1}-\mathrm{DC}}\right)^{(F)_{b}}\right] .
\end{aligned}
$$

Such hierarchies indeed exist and have the intended properties:

LEMMA 7.14. The following is provable in $\left(\mathrm{M}_{\lambda} \uparrow\right)^{\dagger}:$ If $a \triangleleft \lambda<\Phi_{0}$, then

(i) $\left(\exists F \dot{\in} \mathrm{D}_{2 a}\right) \mathrm{Hier} !_{\Sigma_{1}^{1}-\mathrm{DC}}(F, a)$,

(ii) $\forall F, G\left[\operatorname{Hier}_{\Sigma_{1}^{1}-\mathrm{DC}}(F, a) \wedge \operatorname{Hier!}_{\Sigma_{1}^{1}-\mathrm{DC}}(G, a) \rightarrow(\forall b \triangleleft a)(F)_{b}=(G)_{b}\right]$.

Proof. For this proof, we denote claim (i) by $C_{1}(a)$ and claim (ii) by $C_{2}(a)$.

Assume that $a \triangleleft \lambda$. Since $\left\{b \triangleleft a+1:\left(\exists G \dot{\in} \mathrm{D}_{2 b}\right)\right.$ Hier! $\left.!_{\Sigma_{1}^{1}-\mathrm{DC}}(G, b)\right\}$ is a set in $\mathrm{D}_{2(a+1)}$, (i) and (ii) can be shown simultaneously by restricted transfinite induction.

So suppose $c \triangleleft b \triangleleft a+1$ and that $C_{1}(c)$ and $C_{2}(c)$ hold. If $b$ is a successor, then the induction step follows easily form the previous lemma. If $b$ is a limit, then $2 b=b$ and the I.H. yields that

$$
F:=\left\{\langle x, d\rangle: d \triangleleft b \wedge\left(\exists G \dot{\in}\left(\mathrm{D}_{\triangleleft b}\right)_{2(d+1)}\right)\left(\operatorname{Hier}_{\Sigma_{1}^{1}-\mathrm{DC}}(G, d+1) \wedge x \in(G)_{d}\right)\right\}
$$

is an element of $D_{b}$ satisfying Hier! ${ }_{\Sigma_{1}^{\prime}-\mathrm{DC}}(F, b)$, thus $C_{1}(b) . C_{2}(b)$ follows from $C_{1}(b)$ and the I.H.

CoROLlaRY 7.15. For $\alpha<\Phi_{0}$, the following is provable in $\left(\mathrm{M}_{\alpha}+\mathrm{ACA}\right)_{0}^{\dagger}$ :

$$
\exists F \operatorname{Hier}_{\Sigma_{1}^{1}-\mathrm{DC}}(F, \alpha) \text {. }
$$

Proof. $\alpha$ can be written in the form $\omega \cdot \beta+n$ for some $n<\omega$. The claim follows now by (meta-) induction on $n$ : If $n=0$, the previous lemma and arithmetical comprehension yield that

$$
F:=\left\{\langle x, a\rangle: a \triangleleft \omega \cdot \beta \wedge\left(\exists G \dot{\in} \mathrm{D}_{2(a+1)}\right)\left(\operatorname{Hier}_{\Sigma_{1}^{1}-\mathrm{DC}}(G, a+1) \wedge x \in(G)_{a}\right)\right\}
$$

constitutes the sought for hierarchy. If $\left(\mathrm{M}_{\omega, \beta+n}+\mathrm{ACA}\right)_{0}^{\dagger}$ proves the existence of an hierarchy $F$ such that $\operatorname{Hier}_{\Sigma_{1}^{1}-\mathrm{DC}}(F, \omega \cdot \beta+n)$, then $\left(\mathrm{M}_{\omega \cdot \beta+n+1}+\mathrm{ACA}\right)_{0}^{\dagger}$ proves that such a $F$ exists already in $\mathrm{D}_{\omega \cdot \beta+n}$. Then also $(F)_{\triangleleft b} \dot{\in} \mathrm{D}_{\omega \cdot \beta+n}$, and Lemma 7.12 tells us that there exists a model of $\Sigma_{1}^{1}$-DC above $(F)_{\triangleleft b}$ in $\dot{\in} \mathrm{D}_{\omega \cdot \beta+n+1}$. The existence of a hierarchy $G$ satisfying $\operatorname{Hier}_{\Sigma_{1}^{1}-\mathrm{DC}}(G, \omega \cdot \beta+n+1)$ follows.

Next we introduce fixed point hierarchies. For each operator form $\mathscr{A}(\mathrm{P}, \mathrm{Q}, \mathrm{p}, u)$ of $L_{1}(P, Q)$ we say that $H$ constitutes a fixed point hierarchy for $\mathscr{A}$ along $a$ w.r.t. $G$, if

$$
\operatorname{Hier}_{\mathrm{Fix}}^{\mathscr{\alpha}}(G, H, a):=\operatorname{Hier}_{\Sigma_{1}^{1}-\mathrm{DC}}(G, a) \wedge(\forall b \triangleleft a)\left[(H)_{b}=\left(\operatorname{Fix}_{(H)_{\triangleleft b}, b}^{\mathscr{A}}\right)^{(G)_{b}}\right],
$$

holds.

LEMMA 7.16. For $\alpha<\Phi_{0},\left(\mathrm{M}_{\alpha}+\mathrm{ACA}\right)_{0}^{\dagger}$ proves:

(i) $a \unlhd \alpha \wedge \operatorname{Hier}_{\Sigma_{1}^{1}-\mathrm{DC}}(G, \alpha) \rightarrow \exists ! H \operatorname{Hier}_{\mathrm{Fix}}^{\mathscr{d}}(G, H, a)$,

(ii) $\operatorname{Hier}_{\mathrm{Fix}}^{\mathscr{\alpha}}(G, H, \alpha) \wedge \operatorname{Hier}_{\mathrm{Fix}}^{\mathscr{\alpha}}\left(G, H^{\prime}, \alpha\right) \rightarrow(\forall a \triangleleft \alpha)\left[(H)_{a}=\left(H^{\prime}\right)_{a}\right]$,

(iii) $\operatorname{Hier}_{\mathrm{Fix}}^{\mathscr{\alpha}}(G, H, \alpha) \rightarrow(\forall a \triangleleft \alpha)\left[(H)_{\triangleleft a} \dot{\in}(G)_{a}\right]$. 
Proof. Transfinite induction and the definition of the fixed point hierarchy yields immediately the uniqueness assertion (ii). For (i), we assume that $G$ satisfies $\operatorname{Hier}_{\mathrm{Fix}}^{\mathscr{A}}(G, \alpha)$, and show by induction that for each $a \triangleleft \alpha$,

$$
\left(\exists ! H \dot{\in}(G)_{a}\right) \operatorname{Hier}_{\mathrm{Fix}}^{\mathscr{A}}\left((G)_{\triangleleft a}, H, a\right) .
$$

We just consider the successor case, the limit case is similar. So assume that there exists a unique $H \dot{\in}(G)_{b}$ such that $\operatorname{Hier}_{\mathrm{Fix}}^{\mathscr{A}}\left((G)_{\triangleleft b}, H, b\right)$. With $H \dot{\in}(G)_{b}$ we have also $(H)_{\triangleleft b} \dot{\epsilon}(G)_{b}$, which implies that $H^{\prime}:=\left(\operatorname{Fix}_{(H)_{\triangleleft b}, b}^{\mathscr{A}}\right)^{(G)_{b}}$ is an element of $(G)_{b+1}$. Now we obtain a fixed point hierarchy for $\mathscr{A}$ along $b+1$. Claim (iii) follows from (i) and the definition of $\operatorname{Hier}_{\mathrm{Fix}}^{\mathscr{A}}(G, H, a)$.

Lemma 7.14 gives way to the following corollary.

COROLLARY 7.17. For a limit ordinal $\lambda<\Phi_{0},\left(\mathrm{M}_{\lambda} \uparrow\right)^{\dagger}$ proves:

$$
(\forall a \triangleleft \lambda) \exists ! G \exists ! H\left[\operatorname{Hier}_{\Sigma_{1}^{1}-\mathrm{DC}}(G, a) \wedge \operatorname{Hier}_{\mathrm{Fix}}^{\mathscr{\alpha}}(G, H, a)\right] .
$$

Finally, we see that a fixed point hierarchy $H$ w.r.t. $G$ is indeed suitable for an interpretation of the relation symbols $\mathrm{P}^{\star}$ of $\mid \mathrm{D}_{\alpha}^{*}$ and $\left|\mathrm{D}_{\alpha}^{*}\right|$ :

LEMMA 7.18. Given $\alpha<\Phi_{0}$, then for each operator form $\mathscr{A}$ and each $\Pi_{1}^{1}$ formula $D(U, u)$ of $\mathrm{L}_{2}$, the following is provable in $\left(\mathrm{M}_{\alpha}+\mathrm{ACA}\right)_{0}^{\dagger}:$ If $\operatorname{Hier}_{\mathrm{Fix}}^{\mathscr{A}}(G, H, \alpha)$ holds, then also

(i) $(\forall a \triangleleft \alpha) \forall x\left[x \in(H)_{a} \leftrightarrow \mathscr{A}\left((H)_{a},(H)_{\triangleleft a}, x, a\right)\right]$,

(ii) $(\forall a \triangleleft \alpha)\left[C l^{\infty}\left(\left\{x: D\left((G)_{\triangleleft a}, x\right)\right\}, a\right) \rightarrow(H)_{a} \subseteq\left\{x: D\left((G)_{\triangleleft a}, x\right)\right\}\right]$.

Proof. By Lemma 3.4 we know that ACA proves

$$
\forall x\left[x \in \operatorname{Fix}_{Y, y}^{\mathscr{A}} \leftrightarrow \mathscr{A}\left(\mathrm{Fix}_{Y, y}^{\mathscr{A}}, Y, x, y\right)\right] .
$$

For each $a \triangleleft \alpha$ we have that $(H)_{\triangleleft a} \dot{\epsilon}(G)_{a}$ and $(G)_{a}$ is a model of $\Sigma_{1}^{1}$-AC. Thus we can relativize Lemma 3.4 to $(G)_{a}$ and obtain

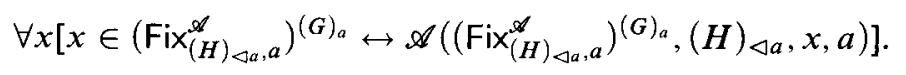

By the definition of $(H)_{a}$ this is equivalent to (i). Claim (ii) is shown analogously by relativizing Theorem 4.1 to $(G)_{a}$.

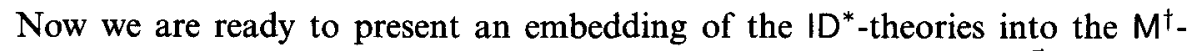
theories: If we have a list $\mathscr{A}_{1}, \ldots, \mathscr{A}_{n}$ of operator forms, then we write $\operatorname{Hier}_{\text {Fix }}^{\overrightarrow{\mathscr{A}^{3}}}(\vec{H}, G, a)$ for

$$
\operatorname{Hier}_{\mathrm{Fix}}^{\mathscr{A}_{1}}\left(H_{1}, G, a\right) \wedge \cdots \wedge \operatorname{Hier}_{\mathrm{Fix}}^{\mathscr{A}_{n}}\left(H_{n}, G, a\right)
$$

An $\mathrm{L}_{\text {Fix }}$ formula $A$ is translated to an $\mathrm{L}_{2}(\mathrm{D})$ formula $A^{*}, A^{\star}$ or $A^{\circ}$ by replacing each subformula of the form $t \in \mathrm{P}^{\mathscr{A}}$, by either the formula $\left(t \in \mathrm{P}^{\mathscr{A}}\right)^{*},\left(t \in \mathrm{P}^{\mathscr{A}}\right)^{\star}$ or $\left(t \in \mathrm{P}^{\mathscr{A}}\right)^{\circ}$, depending on whether we embed $\mathrm{ID}_{\alpha}^{*}$ into $\left.\left(\mathrm{M}_{\alpha}+\mathrm{ACA}\right)_{0}^{\dagger}, \mathrm{ID}_{\alpha+1}^{*}\right\rceil$ into $\left(M_{\alpha}+\Sigma_{1}^{1}-D C\right)_{0}^{\dagger}$ or $I D_{\lambda}^{*}\left\lceil\text { into }\left(M_{\lambda}\right\rceil\right)^{\dagger}$ :

THEOREM 7.19. Let $A$ be an $\mathrm{L}_{\mathrm{Fix}}$ formula that contains exactly the set constants $\mathrm{P}^{\overrightarrow{\mathscr{A}}}$. Then the following holds for each $\alpha<\Phi_{0}$ and each limit $\lambda<\Phi_{0}$ :

$$
\begin{aligned}
& \mathrm{ID}_{\alpha}^{*} \quad \vdash A \Longrightarrow\left(\mathrm{M}_{\alpha}+\mathrm{ACA}\right)_{0}^{\dagger} \vdash \operatorname{Hier}_{\mathrm{Fix}}^{\overrightarrow{\mathrm{f}}}(G, \vec{H}, \alpha) \rightarrow A^{*}, \\
& \mathrm{ID}_{\alpha+1}^{*}\left\lceil\vdash A \Longrightarrow\left(\mathrm{M}_{\alpha}+\Sigma_{1}^{1}-\mathrm{DC}\right)_{0}^{\dagger} \vdash \operatorname{Hier}_{\mathrm{Fix}}^{\vec{*}}(G, \vec{H}, \alpha) \rightarrow A^{\star}\right. \text {, }
\end{aligned}
$$

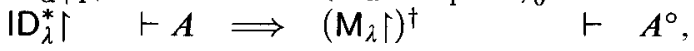


where for $1 \leq i \leq n$,

$$
\begin{aligned}
\left(t \in \mathrm{P}^{\mathscr{A}_{i}}\right)^{*}:= & t=\langle s, a\rangle \wedge a \triangleleft \alpha \wedge t \in H_{i}, \\
\left(t \in \mathrm{P}^{\mathscr{H}_{i}}\right)^{\star}:= & {\left[t=\langle s, a\rangle \wedge a \triangleleft \alpha \wedge s \in\left(H_{i}\right)_{a}\right] \vee\left[t=\langle s, \alpha\rangle \wedge s \in \mathrm{Fix}_{\left(H_{i}\right)_{\triangleleft \alpha}, \alpha}^{\mathscr{A}_{i}}\right], } \\
\left(t \in \mathrm{P}^{\mathscr{A}_{i}}\right)^{\circ}:= & t=\langle s, a\rangle \wedge a \triangleleft \lambda \wedge\left(\exists G, H \in \mathrm{D}_{2(a+2)}\right) \\
& {\left[\operatorname{Hier!} !_{\Sigma_{1}^{1}-\mathrm{DC}}(G, a+1) \wedge\left(\operatorname{Hier}_{\mathrm{Fix}}^{\mathscr{A}_{i}}(G, H, a+1) \wedge s \in(H)_{a}\right)\right] . }
\end{aligned}
$$

Since the existence of the fixed point hierarchies follows form Corollary 7.15 Lemma 7.16 and Corollary 7.17, we also obtain that corresponding theories prove the same $L_{1}$ formulas.

Corollary 7.20. For each $\alpha<\Phi_{0}$, each limit $\lambda<\Phi_{0}$ and each $\mathrm{L}_{1}$ formula $A$, the following holds:

$$
\begin{aligned}
& \mathrm{ID}_{\alpha}^{*} \quad \vdash A \Longrightarrow\left(\mathrm{M}_{\alpha}+\mathrm{ACA}\right)_{0}^{\dagger} \vdash A, \\
& \left|\mathrm{D}_{\alpha+1}^{*}\right| \vdash A \Longrightarrow\left(\mathrm{M}_{\alpha}+\Sigma_{1}^{1}-\mathrm{DC}\right)_{0}^{\dagger} \vdash A \text {, } \\
& \mid \mathrm{D}_{\lambda}^{* \dagger} \vdash A \Longrightarrow\left(\mathrm{M}_{\lambda} \uparrow\right)^{\dagger} \vdash A \text {. }
\end{aligned}
$$

The circle closes: In Section 6 we computed lower bounds for the ID*-theories. In Section 7.1 we proved that these lower bounds are upper bounds for the $M$ theories and thus also for the $\mathrm{M}^{\dagger}$-theories. Eventually, we managed to embed the ID*-theories into the $\mathrm{M}^{\dagger}$-theories. Summing up, we can state the following theorem:

THEOREM 7.21. For each $\alpha<\Phi_{0}$ we have:

$$
\left.|| \mathrm{D}_{\alpha}^{*}|=| \widehat{\mathrm{ID}}_{\alpha} \mid=\sigma(\alpha) \text { and } \quad \mid \mathrm{ID}_{\alpha}^{*}\right\rceil \mid=\sigma \uparrow(\alpha) .
$$

\section{REFERENCES}

[1] Peter Aczel, The strength of Martin-Löf's type theory with one universe, Technical report, Department of Philosophy, University of Helsinki, 1977.

[2] Jeremy AvigAD, On the relationship between $A T R_{0}$ and $\widehat{I D}_{<\omega}$, this JourNAL, vol. 61 (1996), no. 3 , pp. 768-779.

[3] Wilfried Buchiolz, Solomon Feferman, Wolfram Pohlers, and Wilfried Sieg, Iterated inductive definitions and subsystems of analysis: Recent proof-theoretical studies, Lecture Notes in Mathematics, vol. 897, Springer, 1981.

[4] ANDREa CANTINI, A note on a predicatively reducible theory of iterated elementary induction, Bollettino Unione Mathematica Italiana, vol. 4-B (1985), no. 6, pp. 413-430.

[5] - On the relationship between choice and comprehension principles in second order arithmetic, this JourNaL, vol. 51 (1986), pp. 360-373.

[6] Solomon Feferman, Iterated inductive fixed-point theories: Application to Hancock's conjecture, The Patras Symposion (G. Metakides, editor), North Holland, 1982, pp. 171-196.

[7] - Reflecting on incompleteness, this JouRNAL, vol. 56 (1991), no. 1, pp. 1-49.

[8] HARVEY FrIEDMAN, Theories of inductive definition, 1969, Unpublished notes.

[9] Gerhard JäGer, Reinhard Kahle, Anton Setzer, and Thomas Strahm, The proof-theoretic analysis of transfinitely iterated fixed point theories, this JoURNAL, vol. 64 (1999), no. 1, pp. 53-67.

[10] Gerhard JÄGer and Dieter Probst, Variation on a theme of Schütte, Mathematical Logic Quarterly, vol. 50 (2004), no. 3, pp. 258-264.

[11] G. KREISEL, Mathematical Logic, Lectures on modern mathematics (Thomas Lorie Saaty, editor), vol. III, Wiley, 1965, pp. 95-195.

[12] Wolfram Pohlers, Proof Theory: An Introduction, Lecture Notes in Mathematics, vol. 1407, Springer, 1989. 
[13] Dieter Probst, On the relationship between fixed points and iteration in admissible set theory without foundation, Archive for Mathematical Logic, (2005), no. 44, pp. 561-580.

[14] , Pseudo-hierarchies in admissible set theories without foundation and explicit mathematics, Ph.D. thesis, Universität Bern, 2005.

[15] CHRISTIAN RÜEDE, The proof-theoretic analysis of $\Sigma_{1}^{1}$ transfinite dependent choice, Annals of Pure and Applied Logic, vol. 121 (2003), no. 1, pp. 195-234.

[16] KuRT Schütte, Proof Theory, Springer, 1977.

[17] Helmut Schwichtenberg, Proof theory: Some applications of cut-elimination, Handbook of Mathematical Logic (J. Barwise, editor), North Holland, 1977, pp. 867-895.

[18] Stephen G. Simpson, Subsystems of Second Order Arithmetic, Perspectives in Mathematical Logic, Springer, 1998.

\section{UNIVERSITÄT BERN}

INSTITUT FÜR INFORMATIK UND ANGEWANDTE MATHEMATIK

NEUBRÜCKSTRASSE 10, CH-3012 BERN, SWITZERLAND

E-mail:probst@iam.unibe.ch 